\title{
Indeterminacy of Cournot-Walras equilibrium with incomplete markets
}

\section{Michael Zierhut ${ }^{1}$ (D)}

Received: 3 November 2018 / Accepted: 18 January 2020 / Published online: 29 January 2020

(c) The Author(s) 2020

\begin{abstract}
This paper studies a sole proprietorship economy with imperfect competition in a transferable utility setting. While consumers behave as price takers, producers issue real assets strategically to maximize their own utility. Even when complete markets are technologically feasible, equilibria with incomplete markets are robust, and they appear in large numbers: There is a continuum of equilibria with different asset spans that can be welfare-ranked. This real indeterminacy does not vanish as the number of producers goes to infinity. Therefore, the self-interest of producers restricts economic outcomes but does not determine them.
\end{abstract}

Keywords Incomplete markets with production - Imperfect competition · Discontinuous games $\cdot$ Real indeterminacy $\cdot$ Endogenously incomplete markets

JEL classification D43 - D51 · D52

\section{Introduction}

In the Walrasian theory of general economic equilibrium, firms behave as price takers and there is no strategic interaction. When markets are complete and the number of firms is large, the assumption of price-taking behavior is well-founded: Gabszewicz and Vial (1972) start with a small number of firms and enlarge the economy by means of replication. Even though firms are Cournot competitors, their market power vanishes

\footnotetext{
The author thanks Beth Allen, Egbert Dierker, Michael Greinecker, Leonidas Koutsougeras, Klaus Ritzberger, Alex Stomper, Walter Trockel, Jan Werner, Bill Zame, an anonymous referee, as well as participants in the VI Hurwicz Workshop on Mechanism Design Theory, the XXVI European Workshop on General Equilibrium Theory, and the 2017 SAET Conference, for many valuable comments. This research was funded by the German Research Foundation (DFG) under project ZI 1673/1-1.
}

Michael Zierhut

michael.zierhut@hu-berlin.de

1 Institute of Financial Economics, Humboldt University, Dorotheenstr. 1, 10117 Berlin, Germany 
as they grow large in number and small in size relative to the economy. The limit of Cournot competition is a Walrasian equilibrium. The purpose of the present paper is to relax the assumption of complete markets. The setting is otherwise equivalent: It combines a Walrasian consumer side with producers who compete in a Cournot oligopoly, hence the name Cournot-Walras equilibrium. Although the modification seems small, the nature of the equilibrium set changes entirely. This challenges the positive foundations of price-taking behavior.

The model of production is simple: Each firm consists of a production technology, an entrepreneur who makes the decisions, and a group of capitalists who provide outside finance. Following the tradition of general equilibrium, the model is closed and capitalists are consumers driven by their own preferences. For simplicity, there are only two dates and a single good that represents income today and in each future state of the world. Production is financed today by issuing assets in the financial market. Each asset is a claim to a share of the future output. Consumers invest in assets because their portfolios determine their state-contingent future income. A model of this kind is introduced by Carvajal et al. (2012), who choose to make the following two assumptions: First, all agents have quasilinear utility functions. Second, entrepreneurs consume only at the present date. Even though these assumptions are restrictive, they have the merit of rendering the model tractable, with a clear division of roles into consumers and producers, as in the archetypical model of Cournot (1838). ${ }^{1}$

Producers choose their asset payoffs strategically. Since they are entrepreneurs who only care about present income, profit maximization is their natural objective. Quasilinear utility guarantees a single-valued mapping from choices to market prices. The asset payoffs determine not only how income transfers are priced, but also which income transfers are possible in the first place. Ever since Hart (1975) made a broad audience aware of the issue, it is widely understood that demand and prices exhibit discontinuities when the dimension of the income transfer space changes. Discontinuous prices result in discontinuous profits, and discontinuous payoff functions of profit-maximizing producers result in a discontinuous game. So far, little is known about the equilibrium set in such models-neither about its qualitative properties, nor about conditions on the primitives of the model (i.e., preferences, endowments, technologies) that guarantee its nonemptiness. The focus of the present paper is on qualitative properties. The properties of interest are the number of equilibria, the types of assets issued, economic welfare, and the limits of competition as the number of producers grows large.

A classical result due to Dierker (1972) and Harsanyi (1973) is that Walrasian exchange economies as well as mixed extensions of finite games have an odd number of equilibria, provided that certain regularity conditions are fulfilled. It is known that discontinuities violate these conditions, and there is indeed no reason to presume that the number of Cournot-Walras equilibria with incomplete markets is odd. One may be tempted to believe that if the number is not odd, then it should be even, but such intuition is wrong. In the presence of discontinuities, the number of equilibria does not become even; it becomes very large: When markets are endogenously incomplete,

\footnotetext{
${ }^{1}$ It should be noted that the idea of extending the concept of Cournot-Walras equilibrium to asset market economies already appears in Faias (2008).
} 
there is a continuum of Cournot-Walras equilibria. The more incomplete a market becomes, the larger the set of equilibria grows. Denote by $r$ the number of nonredundant assets, and by $|\boldsymbol{\Omega}|$ the number of states; then, $|\boldsymbol{\Omega}|-r$ measures the degree of market incompleteness. Conditional on the existence of at least one equilibrium with $r$ nonredundant assets and a suitable regularity condition, the following results are derived:

\section{There is a real indeterminacy of dimension $r(|\boldsymbol{\Omega}|-r)$ (Theorem 1).}

Only if markets are endogenously complete, i.e., $|\boldsymbol{\Omega}|=r$, Cournot-Walras equilibria are determinate. In all other cases, there is a real indeterminacy in addition to the usual financial indeterminacy that arises in the presence of redundant assets. It includes a continuum of equilibria with different asset spans and different allocations. This result is obtained in a two-date finance economy with real assets. It is therefore in contrast to real indeterminacy found in other settings, such as economies with nominal assets, as in Balasko and Cass (1989), Geanakoplos and Mas-Colell (1989), Werner (1990) and Nagata (1998), or economies with future spot markets, as in Mas-Colell (1991). Since income transfers are restricted to the asset span, a natural question is whether Cournot competition precludes certain types of income transfers. The answer is negative:

2. The set of equilibrium asset spans is open in the space of all $r$-dimensional asset spans (Corollary 2).

This result is interpreted as follows: If there is one equilibrium with an $r$ dimensional asset span, then any nearby asset span (in the space of all $r$-spans) occurs endogenously at another equilibrium. Therefore, a large number of asset structures may arise from Cournot competition. The quasilinear setting lends itself to welfare comparisons of different equilibria. Given the large multiplicity of asset spans and allocations, it is no surprise that different equilibria are not equally desirable from a normative viewpoint:

3. Every open subset of the equilibrium set contains two equilibria that can be welfareranked (Proposition 1).

This leaves room for normative theory: It is possible to design welfare-oriented objectives for firms that are consistent with the self-interest of their owners. The attainable welfare standard is lower than constrained efficiency, but it cannot be raised any further: As Zierhut (2017) shows, constrained efficient production plans would generically be rejected by majority vote of the owners of the firm.

Finally, it is shown that in a world with incomplete markets, the limit behavior of Cournot-Walras equilibrium is different from its complete-market counterpart. The classical result that Walras equilibria are the unique limit points of Cournot competition as economies grow large is no longer true under endogenous asset structure choice:

4. The limit economy, as the number of producers goes to infinity, may have a continuum of incomplete-market equilibria that can be welfare-ranked.

Even though these results reveal several notable properties, they certainly do not constitute an exhaustive analysis of general equilibrium with imperfect competition. 
Any economic model is a compromise between generality and tractability, and setting the focus on one aspect is synonymous with neglecting others. However, several of these neglected aspects are studied elsewhere. The most important one is existence of equilibrium, which is addressed in the growing literature on pure-strategy Nash equilibria in discontinuous games, albeit so far limited to conditions on derived objects (i.e., demand, best replies). ${ }^{2}$ Conditions on the primitives of the model are formulated in existence theorems for Cournot-Walras equilibrium with complete markets, such as in Shirai (2010). However, these results are not applicable in the present setting with an endogenous asset span.

Other neglected aspects include market entry and strategic takeovers of firms. A model with free entry is studied by Novshek and Sonnenschein $(1978,1983)$ in a setting with complete markets. In the models of Milne and Ritzberger (2002) and Demichelis and Ritzberger (2011), production technologies are owned by corporations and large investors acquire shares strategically. Another strand of the literature deals with complications that occur when the assumptions of the present model are relaxed. For example, the mapping from production plans to prices need not be single-valued outside the quasilinear setting. Then, as shown by Dierker and Grodal (1986), existence may fail even in the mixed extension. Moreover, equilibrium in pure strategies can only be defined with respect to a price selection. For discussions of this topic, the reader is referred to Roberts (1980) and Allen (1994).

If there are multiple goods per state, the profit functions of producers depend on the chosen price normalization rule. In an example by Böhm (1994), any allocation can be attained at a Cournot-Walras equilibrium under some normalization rule. Moreover, if a firm has multiple owners who consume multiple goods, there may be disagreement about the optimal production plan, and profit maximization is no longer a well-founded objective of the firm. Dierker and Grodal (1999) and Bejan (2008) attempt to resolve these issues by proposing different objectives of the firm that depend only on relative prices. Nevertheless, as long as the objective function depends on prices, demand, or the utility of consumers, discontinuities arise when the asset structure is endogenous. Therefore, even in a much more general setting one can expect indeterminacy results similar to Theorem 1, but also considerable side effects.

The remainder of this paper is organized as follows: Section 2 presents the model. Section 3 explains the intuition by means of a simple example. Section 4 quantifies the degree of indeterminacy. Section 5 shows that different equilibria can be welfareranked and discusses the normative implications. Section 6 replicates the economy and studies the limit points of Cournot competition in an example. Section 7 concludes.

\section{Model}

Consider a two-date finance economy with a finite number of consumers and a finite number of producers. Production takes time: The input is required at the present date (date 0 ); the output becomes available at the future date (date 1 ). The future is uncertain, and the output quantity depends on the state of the world. At date 0 , producers choose

\footnotetext{
2 See Reny (2016) for a comprehensive survey of this literature.
} 
their production plans. Each producer sells a claim to his state-dependent output in the form of a financial asset. Consumers trade these assets in the financial market. At date 1 , all assets pay off.

The following notation is used throughout: If $x$ is a vector in Euclidean space, $x \geq 0$ means all components are nonnegative, $x>0$ means at least one component is greater than zero, and $x \gg 0$ means all components are greater than zero. The inner product is written as $x \cdot y$, and $\boldsymbol{I}$ stands for the identity matrix. Moreover, for any set $S, \operatorname{id}_{S}$ denotes the identity map on $S$, and if $S$ is a component of a product space, $\operatorname{pr}_{S}$ denotes the projection onto $S$. Prices and gradients are viewed as row vectors, while all other variables are viewed as column vectors.

For a $C^{s}$ function $f: X \rightarrow Z$ between two $C^{s}$ manifolds $X, Z$ with $s \geq 1$, $d f[x]: T_{X}[x] \rightarrow T_{Z}[z]$ denotes the differential of $f$ at $x \in X$, which is a linear function between the tangent spaces $T_{X}[x]$ at $x$ and $T_{Z}[z]$ at $z$. It can be represented in local coordinates by the Jacobian matrix $D f[x]$. For a $C^{s}$ function $f: X \rightarrow \mathbb{R}$ with $s \geq 2, d^{2} f[x]: T_{X}[x] \times T_{X}[x] \rightarrow \mathbb{R}$ denotes the second-order differential, which is the bilinear form whose local representation is the Hessian matrix $D^{2} f[x]$. If $S \subset X$, then $\left.f\right|_{S}$ denotes the domain restriction of $f$ to $S$. If $S \not \subset X$, then $\left.f\right|_{S}$ is implicitly understood as the domain restriction of $f$ to $S \cap X$. Moreover, if $S$ is a closed set, the phrase $f$ is of class $C^{s}$ on $S$ is understood as $\left.f\right|_{S}$ having a $C^{s}$ Whitney extension.

\subsection{Commodities, uncertainty and markets}

There is a single input good at date 0 , which serves as the numéraire. The uncertainty at date 1 is represented by a finite state space $\boldsymbol{\Omega}$. There is one output good for each state of the world. The financial market opens only at date $0: K \geq 2$ assets are traded at prices $p \in \mathbb{R}^{K}$. Their state-dependent payoffs at date 1 are collected in an $|\boldsymbol{\Omega}| \times K$ payoff matrix $\boldsymbol{A}$. It is further assumed that $K \geq|\boldsymbol{\Omega}|$; that is, there are sufficiently many producers such that complete markets are always technologically feasible. There are no short-sale constraints.

\subsection{Consumers}

There are $I \geq 1$ consumers, indexed by superscripts $i \in\{1, \ldots, I\}$. All consumers have identical consumption sets $\mathcal{C}^{i}=\mathbb{R}_{+}^{|\boldsymbol{\Omega}|+1}$. The consumption preferences of consumer $i$ are represented by a utility function $U^{i}: \mathcal{C}^{i} \rightarrow \mathbb{R}$. The endowment of the consumer is $e^{i} \in \mathcal{C}^{i}$. Whenever consumer-specific variables are joined in a single vector, the superscript is omitted; e.g., $e=\left(e^{1}, \ldots, e^{I}\right)$. Whenever consumer-specific variables are aggregated, a bar is put on top; e.g., $\bar{e}=\sum_{i=1}^{I} e^{i}$. Consumers behave as price takers: Each consumer $i$ chooses a consumption plan $c^{i} \in \mathcal{C}^{i}$ and a portfolio $\psi^{i} \in \mathbb{R}^{K}$ from his budget correspondence $B^{i}: \mathbb{R}^{|\boldsymbol{\Omega}| \times K} \times \mathbb{R}^{K} \rightrightarrows \mathcal{C}^{i} \times \mathbb{R}^{K}$, which is defined as

$$
B^{i}(\boldsymbol{A}, p)=\left\{\left(c^{i}, \psi^{i}\right) \in \mathcal{C}^{i} \times \mathbb{R}^{K} \mid c^{i} \leq e^{i}+\left(\begin{array}{c}
-p \\
\boldsymbol{A}
\end{array}\right) \cdot \psi^{i}\right\} .
$$


The asset demand correspondence $\Psi^{i *}: \mathbb{R}^{|\boldsymbol{\Omega}| \times K} \times \mathbb{R}^{K} \rightrightarrows \mathbb{R}^{K}$ maps tuples $(\boldsymbol{A}, p)$ of payoffs and prices to solutions of the utility maximization problem

$$
\max _{c^{i}, \psi^{i}} U^{i}\left(c^{i}\right) \text { subject to }\left(c^{i}, \psi^{i}\right) \in B^{i}(\boldsymbol{A}, p) .
$$

Demand of all consumers is joined in the correspondence $\Psi^{*}=\left(\Psi^{1 *}, \ldots, \Psi^{I *}\right)$.

\subsection{Producers}

There are $K \geq 2$ producers, indexed by superscripts $k \in\{1, \ldots, K\}$. The production set of each producer $k$ is decomposed into a choice set $\mathcal{A}^{k} \subset \mathbb{R}_{+}^{|\boldsymbol{\Omega}|}$, which contains all feasible output vectors, and a cost function $\kappa^{k}: \mathcal{A} \rightarrow \mathbb{R}_{+}$, in which $\mathcal{A}=\chi_{k=1}^{K} \mathcal{A}^{k}$. Producers do not consume at date 1, and their only concern is present profits. Each producer $k$ sells the entire output as an asset with payoffs $A^{k} \in \mathcal{A}^{k}$. The production preferences of producer $k$ are represented by a profit function $\Pi^{k}: \mathcal{A} \times \mathbb{R}_{+}^{K} \rightarrow \mathbb{R}$, defined as revenue minus costs:

$$
\Pi^{k}(\boldsymbol{A}, p)=p^{k}-\kappa^{k}(\boldsymbol{A})
$$

Producers behave strategically: They choose their plans conditional on an inverse demand function $p^{*}: \mathcal{A} \rightarrow \mathbb{R}^{K}$. The payoff function of producer $k$ can be written as

$$
\Pi^{k *}(\boldsymbol{A})=\Pi^{k}\left(\boldsymbol{A}, p^{*}(\boldsymbol{A})\right)
$$

and depends on strategy combinations $\boldsymbol{A}=\left(\boldsymbol{A}^{k}, \boldsymbol{A}^{\neg}\right)$. In this tuple, $\boldsymbol{A}^{k}$ represents his own choice, whereas the collection $A^{\neg k}$ represents the choices of the other $K-1$ producers. The best-reply correspondence $A^{k *}: \mathcal{A} \rightrightarrows \mathcal{A}^{k}$ of producer $k$ associates with each candidate strategy combination $\boldsymbol{A}_{\#} \in \mathcal{A}$ the solution set to his profit maximization problem

$$
\max _{\boldsymbol{A}^{k}} \Pi^{k *}\left(\boldsymbol{A}^{k}, \boldsymbol{A}_{\#}^{\neg k}\right) \quad \text { subject to } \quad \boldsymbol{A}^{k} \in \mathcal{A}^{k} .
$$

\subsection{Economies}

The economy is defined by the characteristics of its consumers and producers. Consumers are described by their utility functions and endowments, which satisfy the following assumptions.

Assumption 1 (Preferences) For each consumer $i$,

$$
U^{i}\left(c^{i}\right)=c_{0}^{i}+u^{i}\left(c_{1}^{i}\right)
$$

for some function $u^{i}: \mathbb{R}_{+}^{|\boldsymbol{\Omega}|} \rightarrow \mathbb{R}$ that satisfies for each $c_{\mathbf{1}}^{i} \in \mathbb{R}_{++}^{|\boldsymbol{\Omega}|}$, 
1. $u^{i}$ is continuous and of class $C^{3}$ on $\mathbb{R}_{++}^{|\boldsymbol{\Omega}|}$

2. $d u^{i}\left[c_{\mathbf{1}}^{i}\right](v) \gg 0 \forall v>0$

3. $d^{2} u^{i}\left[c_{1}^{i}\right](v, v)<0 \forall v \neq 0$

4. $d u^{i}\left[c_{\mathbf{1}}^{i}\right]\left(\boldsymbol{I}_{\omega}\right) \rightarrow \infty$ as $c_{\omega}^{i} \rightarrow 0$ for any $\omega \in \boldsymbol{\Omega}$.

Assumption 2 (Endowments) Endowments satisfy $e \gg 0$.

Under Assumption 1, utility is quasilinear in consumption at date 0 and consumers always desire positive consumption at date 1. Under Assumption 2, consumers have a nonzero endowment in all states of the world, which guarantees that the budget correspondence does not exhibit a discontinuity when some price goes to zero. Denote by $\mathbb{E} \subset \mathcal{C}$ the set of endowments that satisfy this assumption. Producers are described by their choice sets and cost functions, which satisfy the following assumptions.

Assumption 3 (Production possibilities) For each producer $k, \mathcal{A}^{k}$ is a convex, compact subset of $\mathbb{R}_{+}^{|\boldsymbol{\Omega}|}$ with nonempty interior.

Assumption 4 (Production costs) For each producer $k, \kappa^{k}$ is continuous and of class $C^{2}$ on the interior of $\mathcal{A}^{k}$.

Note that the interior of $\mathcal{A}^{k}$ is an open subset of $\mathbb{R}^{|\boldsymbol{\Omega}|}$ and therefore a smooth $|\boldsymbol{\Omega}|$ dimensional manifold. Economies are completely defined by the characteristics of consumers and producers.

Definition 1 An economy is a tuple $(U, e, \mathcal{A}, \kappa)$ that satisfies Assumptions $1,2,3$, and 4.

It is implicitly understood that all endogenous objects depend on the economy, and in the interest of a compact notation, economies are omitted as arguments; for example, $\Psi^{*}(\boldsymbol{A}, p)$ is written instead of $\Psi^{*}(\boldsymbol{A}, p, U, e, \mathcal{A}, \kappa)$.

\subsection{Cournot-Walras Equilibrium}

The economy is in equilibrium if all consumers choose portfolios and consumption optimally, all producers play best replies, and prices are such that markets clear. The asset span is the linear subspace $\operatorname{Im}(\boldsymbol{A})$ of feasible transfers of future consumption. If $\operatorname{rank}(\boldsymbol{A})=|\boldsymbol{\Omega}|$, markets are complete and arbitrary transfers are feasible. If $\operatorname{rank}(\boldsymbol{A})<$ $|\boldsymbol{\Omega}|$, markets are incomplete. Denote by $\mathcal{A}_{r}$ the set of asset payoff matrices with rank $r$

$$
\mathcal{A}_{r}=\{\boldsymbol{A} \in \mathcal{A} \mid \operatorname{rank}(\boldsymbol{A})=r\},
$$

and denote by $\mathcal{X}_{r}$ the set of no-arbitrage income transfer matrices for $r$-dimensional asset spans

$$
\mathcal{X}_{r}=\left\{(\boldsymbol{A}, p) \in \mathcal{A}_{r} \times \mathbb{R}_{+}^{K} \mid \operatorname{Im}\left(\begin{array}{c}
-p \\
\boldsymbol{A}
\end{array}\right) \cap \mathbb{R}_{+}^{|\boldsymbol{\Omega}|+1}=\{0\}\right\} .
$$


Then, the budget correspondence $B^{i}$ is continuous on $\mathcal{X}_{r}$ for any choice of $r \in \mathbb{N}$ and has compact-convex values. Continuity of $B^{i}$ fails at those points where the rank of $\left(\begin{array}{c}-p \\ \boldsymbol{A}\end{array}\right)$ changes. Compactness fails in the presence of arbitrage opportunities; that is, if there are portfolios $\psi^{\prime}, \psi^{\prime \prime} \in \mathbb{R}^{K}$ with identical payoffs $\boldsymbol{A} \cdot \psi^{\prime}=\boldsymbol{A} \cdot \psi^{\prime \prime}$ but different prices $p \cdot \psi^{\prime} \neq p \cdot \psi^{\prime \prime} .^{3}$ At all other points, utility maximization problem (2) has a solution. Under Assumption 1, the optimal consumption of consumer $i$ can be expressed as a function $c^{i *}: \mathcal{A} \times \mathbb{R}^{K} \rightarrow \mathcal{C}^{i}$. This function is of class $C^{2}$ on $\mathcal{X}_{r}$ by continuity of $B^{i}$. The supply of all assets is normalized to one, such that a price vector $p$ clears the market if there is some $\psi \in \Psi^{*}(\boldsymbol{A}, p)$ such that

$$
\bar{\psi}=1 \text {. }
$$

As strong monotonicity of $U^{i}$ implies that the budget constraint in (1) holds with equality, the above asset market clearing condition implies that supply and demand of future consumption meet; that is, $\bar{c}_{\mathbf{1}}^{i}=\bar{e}_{\mathbf{1}}^{i}+\overline{\boldsymbol{A}}$. The inverse demand function $p^{*}: \mathcal{A} \rightarrow \mathbb{R}^{K}$ is defined as the solution to

$$
\bar{c}_{\mathbf{1}}^{*}\left(\boldsymbol{A}, p^{*}(\boldsymbol{A})\right)=\bar{e}_{\mathbf{1}}+\overline{\boldsymbol{A}} .
$$

Due to quasilinear utility, there exists a unique function $p^{*}$ that solves (7). ${ }^{4}$ This function is continuous on $\mathcal{A}_{r}$ because $\bar{c}_{1}^{*}$ is continuous on $\mathcal{X}_{r}$. The market for present consumption is implicitly cleared by Walras' law.

Definition 2 A Cournot-Walras equilibrium for economy $(U, e, \mathcal{A}, \kappa)$ with inverse demand $p^{*}$ is a tuple $(\boldsymbol{A}, p, c, \psi)$ such that $\boldsymbol{A}=A^{*}(\boldsymbol{A}), p=p^{*}(\boldsymbol{A}), c=c^{*}(\boldsymbol{A}, p)$, $\psi \in \Psi^{*}(\boldsymbol{A}, p)$, and $\bar{\psi}=\mathbf{1}$.

Note that the present model is a variant of the Cournot-Walras model with financial markets introduced in Zierhut (2020), which differs in the following aspects: On the one hand, the present assumptions on producers are more liberal and permit an endogenous asset span, including endogenously incomplete markets. These are the focus of the present paper. On the other hand, the present assumptions on consumers are more restrictive in that utility is quasilinear. This is a simplification. Without quasilinear utility, there may be multiple solutions to (7) and the equilibrium concept is conditional on a selection $p^{*}$. This selection problem disappears under quasilinear utility, and $p^{*}$ is a unique inverse demand function, as in the early treatment of Gabszewicz and Vial (1972). To study the set of Cournot-Walras equilibria, some degree of differentiability is required at least locally. Equilibria that meet this requirement are called regular.

\subsection{Regularity}

Let $(\boldsymbol{A}, p, c, \psi)$ be a Cournot-Walras equilibrium and denote by $r=\operatorname{rank}(\boldsymbol{A})$ its rank. Let $\psi^{*}$ be a local selection at $(\boldsymbol{A}, p)$ of the demand correspondence $\Psi^{*}$ that is of class

\footnotetext{
${ }^{3}$ For a detailed discussion of arbitrage-free markets the reader is referred to Magill and Quinzii (1996, $\S 9$.$) .$

${ }^{4}$ For a proof of existence and uniqueness, see Hens and Pilgrim (2002), Theorem 6.9, p. 143.
} 
$C^{2}$ on $\mathcal{X}_{r}$. More precisely: $\psi^{*}$ is a selection of $\left.\Psi^{*}\right|_{\mathcal{O}}$ for some open neighborhood $\mathcal{O}$ of $(\boldsymbol{A}, p)$. The existence of such a local selection is proven in Lemma A10 in the appendix. Since the interior of $\mathcal{A}_{r}$ is a smooth manifold, the tangent space $T \mathcal{A}_{r}[\boldsymbol{A}]$ is well-defined.

Definition 3 A Cournot-Walras equilibrium with $\operatorname{rank}(\boldsymbol{A})=r$ is regular if

1. $d_{p} \bar{\psi}^{*} \mid \mathcal{X}_{r}[\boldsymbol{A}, p] \operatorname{spans} \operatorname{Im}\left(\boldsymbol{A}^{\top}\right)$

2. $A^{*}$ is continuously differentiable at $\boldsymbol{A}$

3. $\left.d A^{*}\right|_{\mathcal{A}_{r}}[\boldsymbol{A}]-\operatorname{id}_{T \mathcal{A}_{r}[\boldsymbol{A}]} \operatorname{spans} \operatorname{Im}(\boldsymbol{A})^{K}$.

An equilibrium that is not regular is called critical. If $r=|\boldsymbol{\Omega}|$, then Definition 3 agrees with the concept of regularity introduced in Zierhut (2020), $\psi^{*}$ is continuously differentiable, and in the generic economy all equilibria with rank $r$ are regular. ${ }^{5}$ However, if markets are endogenously incomplete with $r<|\boldsymbol{\Omega}|$, this is no longer true: First, any selection $\psi^{*}$ is discontinuous at $(\boldsymbol{A}, p)$. Second, regular equilibria are typically accompanied by critical equilibria. Both properties will be demonstrated by means of a simple example in Sect. 3. Since discontinuous demand results in discontinuous inverse demand $p^{*}$, payoff functions (4) of producers are discontinuous. Therefore, the Cournot game becomes a discontinuous game.

Consider a restricted game that has the following properties in the neighborhood $\mathcal{O}$ : The payoffs of all producers are of class $C^{2}$, and each equilibrium with $r$-dimensional asset span of the original Cournot game is also an equilibrium of the restricted game (restricted equilibrium for short). The aim is to infer properties of the set $\Xi_{r}$ of (unrestricted) Cournot-Walras equilibria with rank $r$ from the set $\hat{\Xi}_{r}$ of restricted equilibria. To define such a restricted game, consider the correspondence $\hat{\mathcal{A}}_{r}: \mathcal{A}_{r} \rightrightarrows$ $\mathcal{A}_{r}$ with decomposition $\hat{\mathcal{A}}_{r}=\hat{\mathcal{A}}_{r}^{1} \times \cdots \times \hat{\mathcal{A}}_{r}^{K}$ that associate with each reference asset structure $\boldsymbol{A}_{\# \text { the restricted choice sets }}$

$$
\hat{\mathcal{A}}_{r}^{k}\left(\boldsymbol{A}_{\#}\right)=\left\{\boldsymbol{A}^{k} \in \mathcal{A}^{k} \mid \operatorname{rank}\left(\boldsymbol{A}^{k}, \boldsymbol{A}_{\#}^{-k}\right)=r\right\} .
$$

The only modification in the restricted game, relative to the original Cournot game, is that producers solve

$$
\left.\max _{\boldsymbol{A}^{k}} \Pi^{k *}\right|_{\mathcal{A}_{r}}\left(\boldsymbol{A}^{k}, \boldsymbol{A}_{\#}^{\neg k}\right) \quad \text { subject to } \boldsymbol{A}^{k} \in \hat{\mathcal{A}}_{r}^{k}\left(\boldsymbol{A}_{\#}\right) .
$$

instead of (5). By Eq. (4), the payoff function $\left.\Pi^{k *}\right|_{\mathcal{A}_{r}}$ is of class $C^{2}$ on $\mathcal{A}_{r}$, provided that $p^{*}$ has these properties (and Lemma A11 in the appendix verifies that $p^{*}$ has these properties). Let $\Xi_{r}^{*}$ and $\hat{\Xi}_{r}^{*}$ be the sets of regular equilibria of the Cournot game and the restricted game, respectively. While $\Xi_{r} \subset \hat{\Xi}_{r}$ by construction, it may well be that $\Xi_{r}^{*} \nsubseteq \hat{\Xi}_{r}^{*}$. An equilibrium that is regular in the Cournot game need not be regular in the restricted game. At times, it will be convenient to focus on equilibria that are regular in both games and thus belong to the set $\Xi_{r}^{* *}=\Xi_{r}^{*} \cap \hat{\Xi}_{r}^{*}$. These equilibria are called strongly regular.

\footnotetext{
5 In this context, generic means: within an open, dense set of economies. For a discussion and proof, the reader is referred to Zierhut (2020), in particular Corollary 4.
} 
Definition 4 A Cournot-Walras equilibrium with $\operatorname{rank}(\boldsymbol{A})=r$ is strongly regular if it lies in the set $\Xi_{r}^{* *}$; that is, it is a regular equilibrium of both the unrestricted game and the restricted game.

\section{Example}

Consider an economy with $I=2$ consumers, $K=2$ producers, and $|\boldsymbol{\Omega}|=2$ states of the world. Both consumers have identical utility functions

$$
U^{i}\left(c^{i}\right)=c_{0}^{i}+\sum_{\omega \in \Omega} \ln \left(c_{\omega}^{i}\right) \quad i=1,2
$$

but different endowments $e^{1}=(2,0,1 / 2)^{\top}$ and $e^{2}=(2,0,0)^{\top}$. Both producers have identical production technologies with zero costs $\kappa^{k}=0$ and

$$
\mathcal{A}^{k}=\left\{\left(\begin{array}{c}
1 / 2+\alpha^{k} \\
1 / 2-\alpha^{k}
\end{array}\right) \mid \alpha^{k} \in[-1 / 2,1 / 2]\right\} \quad k=1,2,
$$

which are described completely by a single parameter $\alpha^{k}$ each. Therefore, the joint strategy space can be depicted in two dimensions. ${ }^{6}$ The asset structure $A=\left(A^{1}, A^{2}\right)$ is determined endogenously by the producers. Whenever $\alpha^{1} \neq \alpha^{2}, \operatorname{rank}(\boldsymbol{A})=2$ and markets are complete; when $\alpha^{1}=\alpha^{2}$, one asset is redundant, $\operatorname{rank}(\boldsymbol{A})=1$, and markets are incomplete. It should be noted that the demand correspondences $\Psi^{i *}$ are single-valued only if there is no redundant asset. In the case of $\alpha^{1}=\alpha^{2}$, both consumers are indifferent between assets 1 and 2: Both assets have identical payoffs and, by the no-arbitrage principle, identical prices. Therefore, it is possible to define a single-valued selection

$$
\psi^{i *}(\boldsymbol{A}, p)= \begin{cases}\Psi^{i *}(\boldsymbol{A}, p) \cap\left\{\psi^{i} \in \mathbb{R}^{2} \mid \psi_{1}^{i}=\psi_{2}^{i}\right\} & \text { if } \alpha^{1}=\alpha^{2} \\ \Psi^{i *}(\boldsymbol{A}, p) & \text { otherwise }\end{cases}
$$

having the property that $c^{i *}(\boldsymbol{A}, p)$ and $\psi^{i *}(\boldsymbol{A}, p)$ jointly solve utility maximization problem (2). The aggregate demand function $\bar{\psi}^{*}(\boldsymbol{A}, p)=\psi^{1 *}(\boldsymbol{A}, p)+\psi^{2 *}(\boldsymbol{A}, p)$ is of class $C^{2}$ on $\mathcal{X}_{1}$ and $\mathcal{X}_{2}$. Therefore, by the implicit function theorem, the solution $p^{*}$ to the market clearing condition $\bar{\psi}^{*}\left(\boldsymbol{A}, p^{*}(\boldsymbol{A})\right)=\mathbf{1}$ is of class $C^{2}$ on $\mathcal{A}_{1}$ and $\mathcal{A}_{2}$, and so is $\Pi^{*}$.

The best-reply correspondences of both producers are illustrated in Fig. 1. In each panel, the strategy of producer 1 is drawn along the horizontal axis, and the strategy of producer 2 is drawn along the vertical axis. All symmetric strategy combinations $\alpha^{1}=\alpha^{2}$, which result in incomplete markets, lie on the diagonal. Given any $\alpha^{2} \leq$ -0.123 and $\alpha^{2} \geq 0.322$, producer 1 finds it optimal to issue a nonredundant asset and thus to complete the market. At $\alpha^{2}=-0.123$ and at $\alpha^{2}=0.322$, producer 1 is

\footnotetext{
${ }^{6}$ For the sake of tractability, $e$ has some components equal to zero and the interior of $\mathcal{A}^{k}$ is empty, which is not consistent with Assumptions 2 and 3 but innocuous in the present example.
} 

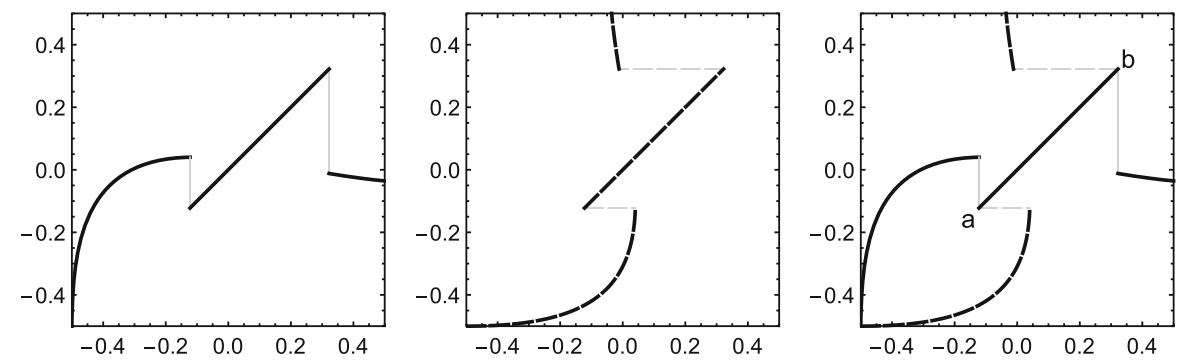

Fig. 1 Best-reply correspondences of Producer 1 (solid) and Producer 2 (dashed) over the space of strategies $\alpha^{1}$ (horizontal axis) and $\alpha^{2}$ (vertical axis)

indifferent between issuing a nonredundant asset and imitating the strategy of producer 2. At these points, his best-reply correspondence exhibits a discontinuity and maps to a set of cardinality two. For all points in between, the best reply of producer 1 lies on the diagonal. Due to the symmetry of the example, the best-reply correspondence of producer 2 has the same shape.

The right panel displays the best-reply correspondences of both producers. It is easy to see that there is a continuum of fixed points: The entire line segment $\overline{a b}$ consists of Cournot-Walras equilibria, each having an asset span of dimension 1. More abstractly, this set of equilibria is a 1-dimensional manifold with boundary. While this set need not be linear in general, it will be verified in Sect. 4 that the manifold structure survives in the general setting. The two boundary equilibria are critical, whereas all other equilibria are regular. Moreover, all regular equilibria are strongly regular because the best-reply correspondence in the restricted game is simply $\hat{A}^{*}=\operatorname{id}_{\mathcal{A}_{1}}$; thus, $\Xi_{1}^{*}=\Xi_{1}^{* *}$.

To understand the source of this multiplicity of equilibria, it is helpful to study the payoff function of one producer. Figure 2 shows the profit of producer 1 for all possible strategies $\alpha^{1}$ in response to a fixed strategy $\alpha^{2}=0.12$ of his competitor. The profit function is discontinuous at those points at which the asset span collapses to a ray. In the figure, this happens at $\alpha^{1}=\alpha^{2}=0.12$, where the profit function exhibits an upward jump. Since this profit is higher than the profit under all other strategies, it is clear that imitation and thus an incomplete asset structure is the best reply of the producer.

If both producers find it optimal to imitate their competitor, which is to say that $\alpha^{1}=\alpha^{2}=0.12$ is indeed an equilibrium, a multiplicity of equilibria is inevitable: There are two local maxima with profits indicated by the horizontal dashed lines. The profit at the global maximum is strictly greater than the profit at the second-best reply. Since $\Pi^{*}$ is of class $C^{2}$ on $\mathcal{A}_{1}$ and $\mathcal{A}_{2}$, there must be neighborhoods $\mathcal{N}_{1} \subseteq \mathcal{A}_{1}$ of the combination of best replies and $\mathcal{N}_{2} \subseteq \mathcal{A}_{2}$ of the combination of second-best replies, such that both producers prefer any strategy combination in $\mathcal{N}_{1}$ over all strategy combinations in $\mathcal{N}_{2}$. Thus, the entire set $\mathcal{N}_{1}$ consists of fixed points of $A^{*}$.

It should be noted that such multiplicity of equilibria with incomplete markets is robust. Consider perturbations of endowments $e$ : By the implicit function theorem, $\left.\Pi^{*}\right|_{\mathcal{A}_{1}}$ and $\left.\Pi^{*}\right|_{\mathcal{A}_{2}}$ vary continuously in some neighborhood $\mathcal{E} \subset \mathbb{E}$ of $e$. By continuity, every endowment vector $e^{\prime} \in \mathcal{E}$ must result in best replies in $\mathcal{A}_{1}$ that are strictly 


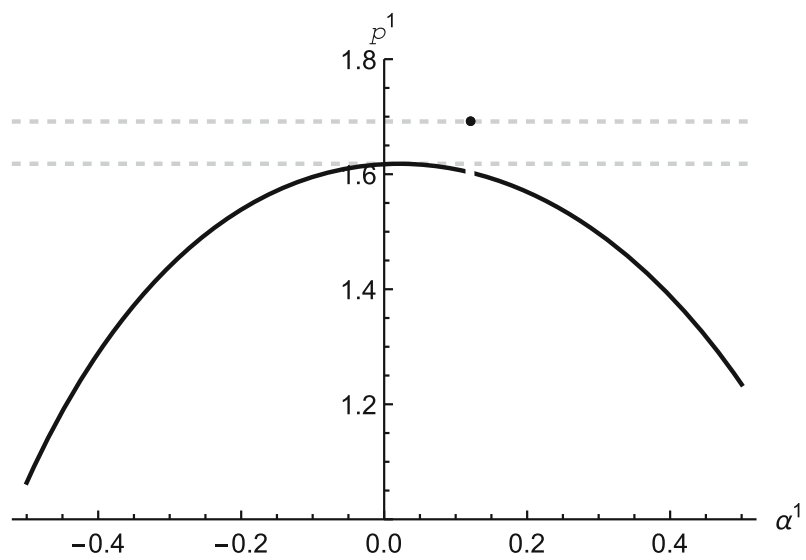

Fig. 2 Profit of Producer 1 as a function of $\alpha^{1}$ for fixed $\alpha^{2}=0.12$

preferred to second-best replies in $\mathcal{A}_{2}$ by both producers. Therefore, discontinuities in the payoff functions of producers have twofold consequences: On the one hand, equilibria with incomplete markets, which always lie right at a discontinuity of $p^{*}$, appear in large numbers. On the other hand, the cardinality of the equilibrium set is robust to perturbations of the economy.

This multiplicity of Nash equilibria in the Cournot game results in real indeterminacy of allocations on the Walrasian side of the model. To analyze whether these different economic outcomes are equally desirable from a normative viewpoint, the transferable utility property is used to measure welfare as

$$
W=u^{1}\left(c^{1}\right)+u^{2}\left(c^{2}\right)=\sum_{i \in\{1,2\}} \sum_{\omega \in \boldsymbol{\Omega}} \ln \left(c_{\omega}^{i}\right) .
$$

Figure 3 displays the welfare values of all equilibria with $\alpha^{1}=\alpha^{2}$ in the interval $\alpha^{k} \in[-0.123,0.322]$. It is easy to see that different equilibria can be welfare-ranked. Moreover, moving toward a welfare-superior equilibrium does not require a strong intervention: At any welfare suboptimal equilibrium, there is small change in production plans that is still consistent with oligopolistic competition but leads to greater transferable utility. Within the set of all equilibrium strategy combinations, the welfare optimum is attained at $\alpha^{1}=\alpha^{2}=0.087$. This is the preferred outcome of a weak social planner who can only redistribute the numéraire commodity once production is finished and the market has closed. Nevertheless, a more powerful planner who can choose production plans before the market opens is still able to achieve a welfare improvement. Any change in production plans of the form $\alpha^{1}+\varepsilon$ for Producer 1 and $\alpha^{2}-\varepsilon$ for Producer 2, with $\varepsilon \neq 0$, leads to complete markets but leaves the aggregate input and output unchanged. Once the market opens, trade results in an optimal distribution of the optimal output. Therefore, a Pareto optimum is reached without any further intervention of the planner. Moreover, since utility is transferable in present consumption, welfare is maximized at the Pareto optimal market outcome. 


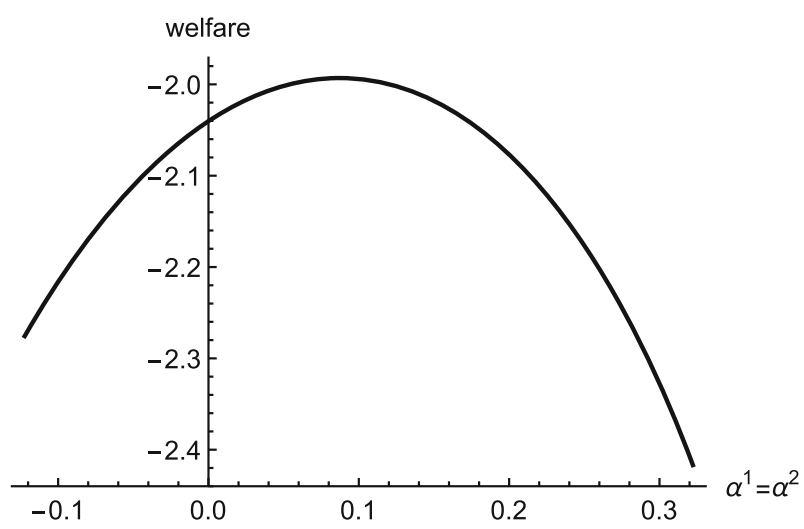

Fig. 3 Welfare of all equilibria on the manifold $\overline{a b}$

Finally, it should be noted that the set of Cournot-Walras equilibria is actually a 2-dimensional manifold with boundary, not a 1-dimensional manifold. Each equilibrium in the line segment $\overline{a b}$ is only unique up to the selection $\psi^{*}$ from the demand correspondence. This selection is to a certain extent arbitrary: Since one of the two assets is redundant at any equilibrium, any change in portfolios of the form $\Delta_{\varepsilon} \psi^{1}=-\Delta_{\varepsilon} \psi^{2}=(\varepsilon,-\varepsilon)$ has no effect on allocations $c$ and therefore satisfies utility maximization problem (2) at the same prices $p$. If $(\boldsymbol{A}, p, c, \psi)$ is an equilibrium, so is $\left(\boldsymbol{A}, p, c, \psi+\Delta_{\varepsilon} \psi\right)$ for any $\varepsilon \in \mathbb{R}$. These equilibria are identical in terms of consumption and welfare but involve different trades in the market for financial assets. This 1-dimensional financial indeterminacy and the 1-dimensional real indeterminacy sum up to an equilibrium set of dimension 2 .

\section{Indeterminacy}

The degree of financial indeterminacy is always easy to compute: The only source of such indeterminacy is redundant assets, and each feasible transfer can be realized by $K-r$ portfolios. The rest is simple algebra: There are $I$ consumers who must choose their portfolios. However, once the first $I-1$ consumers have made their choices, the portfolio of the final consumer is determined by the market clearing condition. This results in financial indeterminacy of degree $(I-1)(K-r)$. Contrary to financial indeterminacy, which is welfare-neutral, real indeterminacy involves different allocations and is therefore welfare-relevant. To quantify the degree of real indeterminacy, the best-reply correspondence must be studied. This problem is approached in two steps: In the first step, the set of strongly regular equilibria is described. It turns out that this set is particularly well behaved. It has the structure of a differentiable manifold. In the second step, this structure is used to deduce the dimension of the larger set of regular equilibria. Step one: 
Theorem 1 For any $r \in \mathbb{N}$, the set of strongly regular equilibria with $r$-dimensional asset span, $\Xi_{r}^{* *}$, is a $C^{1}$ manifold of dimension $r(|\boldsymbol{\Omega}|-r)+(I-1)(K-r)$ or empty. The degree of real indeterminacy is $r(|\boldsymbol{\Omega}|-r)$.

The proof of Theorem 1 is based on the differential topology of vector bundles. The reader unfamiliar with this topic will find a concise summary of all necessary concepts in the appendix, before the proof of the theorem is presented in full detail. ${ }^{7}$ The logic of the proof can be outlined as follows: If $(\boldsymbol{A}, p, c, \psi)$ is a Cournot-Walras equilibrium with $\operatorname{rank}(\boldsymbol{A})=r$, then for each producer $k, \Pi^{k *}\left(\boldsymbol{A}^{k}, \boldsymbol{A}^{-k}\right) \geq \Pi^{k *}\left(\boldsymbol{A}_{\#}^{k}, \boldsymbol{A}^{\neg k}\right)$ for any $\boldsymbol{A}_{\#}^{k}$ that leads to a different rank. If the equilibrium is strongly regular, the inequality is strict and $\Xi_{r}^{* *}$ is open in $\hat{\Xi}_{r}^{*}$. Moreover, strong regularity implies that restricted equilibria can be described as the zeroes of a $C^{1}$ function between vector bundles. The set $\hat{\boldsymbol{\Xi}}_{r}^{*}$ is the preimage of the zero section under this function, and its dimension is determined by means of the preimage theorem.

This result can be extended to the whole set $\Xi_{r}^{*}$ of regular equilibria. It turns out that $\Xi_{r}^{* *}$ and $\Xi_{r}^{*}$ are of the same dimension, even though the latter need not have the structure of a $C^{1}$ manifold. Step two:

Corollary 1 If $\Xi_{r}^{* *}$ is nonempty, the set of regular equilibria $\Xi_{r}^{*}$ is of dimension $r(|\boldsymbol{\Omega}|-$ $r)+(I-1)(K-r)$. The degree of real indeterminacy is $r(|\boldsymbol{\Omega}|-r)$.

In light of this quantitative result, which reveals that a large set of asset structures may occur in equilibrium, a natural qualitative question is what kind of asset can and what kind cannot arise in equilibrium. To address this question, one further corollary is added:

Corollary 2 If $\Xi_{r}^{* *}$ is nonempty, the set of $r$-dimensional equilibrium asset spans is open in the set of all $r$-dimensional asset spans.

These results reveal a stark contrast of economic outcomes between oligopolies with complete and incomplete markets. When markets are complete, the self-interest of producers determines the economic outcome. In this case, $|\boldsymbol{\Omega}|=r$ and thus there is no real indeterminacy. In addition, if $K=|\boldsymbol{\Omega}|$ as in the example, there are no redundant assets, i.e., $K=r$, which eliminates the financial indeterminacy. As a consequence, $\Xi_{r}^{*}$ is a finite set of isolated equilibria by Corollary 1 .

When markets are incomplete, a large set of outcomes are all individually rational for producers. The dimension of this set grows with the degree of market incompleteness: A decrease in the dimension of the asset span $r$ has two effects. On the one hand, the degree of market incompleteness $|\boldsymbol{\Omega}|-r$ becomes greater, which enlarges the real indeterminacy. On the other hand, the number of redundant assets $K-r$ increases, which enlarges the financial indeterminacy. While individual rationality is a very restrictive requirement for production decisions when markets are complete, it permits a rich variety of asset structures in incomplete markets.

Corollary 2 goes one step further and shows that locally anything goes: No asset span can be ruled out a priori. Recall that the set of all asset spans of a given dimension

\footnotetext{
7 The idea of using vector bundles to model incomplete market economies goes back to Husseini et al. (1990) and Hirsch et al. (1990).
} 
is known to be a manifold called the Grassmannian. The set of asset spans that can occur in equilibrium is a submanifold. The difference in dimension between the two manifolds measures how restrictive the self-interest of producers really is. As the corollary shows: not at all. At a regular Cournot-Walras equilibrium with incomplete markets, the asset span can be tilted in any direction, and provided that it is not tilted too far, there exists another regular equilibrium with exactly that new asset span.

These findings are not limited to strategic production choices but extend to all kinds of financial innovation that cause a discontinuity in demand. Consider the baseline model of Carvajal et al. (2012) in which a predetermined production plan is split and sold in the form of multiple assets. Even though the choice sets of such producers are different, they still act as financial innovators when they issue nonredundant assets. Such financial innovation causes a discontinuity in their payoff functions, and the result is again a multiplicity of equilibria. Each of the resulting equilibria is perfectly consistent with the self-interest of producers, but contrary to Walrasian economies this self-interest restricts possible economic outcomes to a set rather than to a single allocation. Given this abundance of possible economic outcomes, it should not be surprising that a nontrivial welfare ranking is possible. This is formally proven in Sect. 5.

\section{Welfare}

Since utility is linear in consumption at date 0 for consumers and producers alike, there is a natural welfare function $W: \mathcal{A} \times \mathbb{R}^{K} \times \mathcal{C} \times \mathbb{R}^{I K} \rightarrow \mathbb{R}$ that can be used to compare different equilibria:

$$
W(\boldsymbol{A}, p, c, \psi)=\sum_{i=1}^{I} u^{i}\left(c_{\mathbf{1}}^{i}\right)-\sum_{k=1}^{K} \kappa^{k}\left(\boldsymbol{A}^{k}\right)
$$

The function $W$ can be interpreted as the objective function of a social planner who aims at Pareto efficiency, at least in a constrained sense. As utility is transferable, there is no conflict between efficiency and welfare, and the Pareto frontier coincides with the set of maxima of $W .{ }^{8}$ Consider the weakest possible social planner: This planner can make no transfers other than a redistribution of date 0 consumption after the market has closed. The planner can only propose production plans to all producers, but they are free to deviate if they dislike the proposal. If $W$ attains a maximum $\xi^{*}$ on $\Xi_{r}^{* *}$ with production plans $\boldsymbol{A}$, then these are the production plans the planner would propose.

Note that $W$ defines a total order in the ambient space of the equilibrium manifold. In the example from Sect. 3, $\Xi_{r}^{* *}$ is a nontrivially ordered set; that is, there are at least two equilibria that are not welfare-equivalent. In fact, the example satisfies a stronger property: Any open subset of $\Xi_{r}^{* *}$ is a nontrivially ordered set. Therefore, at any suboptimal equilibrium, a slight intervention of the social planner can already attain a Pareto improvement. The following proposition establishes this as a generic

\footnotetext{
${ }^{8}$ In production economies without transferable utility, these two concepts are not well aligned; see Dierker and Dierker (2010).
} 
property. The set $\Xi_{r}^{* *}$ of strongly regular equilibria with rank $r$ is said to admit $a$ locally nontrivial welfare ranking if any open subset of $\Xi_{r}^{* *}$ contains two equilibria that can be welfare-ranked. The proof of the following proposition can be found in the appendix:

Proposition 1 For any fixed $(U, \mathcal{A}, \Pi)$ there is an open, dense subset $\mathbb{E}_{*} \subseteq \mathbb{E}$ having full Lebesgue measure such that $\Xi_{r}^{* *}$ admits a locally nontrivial welfare ranking for any economy $(U, e, \mathcal{A}, \Pi)$ with $e \in \mathbb{E}_{*}$ and any $r \in \mathbb{N}$ such that $r<|\boldsymbol{\Omega}|$.

The geometric interpretation of Proposition 1 is rather simple: Even though the equilibrium manifold is small enough to be contained in an isoquant of the welfare function, this happens only in a negligible set of economies. More important is the economic interpretation of this result: Typically, some equilibria are socially more desirable than others. Since all equilibria are individually rational for all producers, even a very weak social planner, who cannot enforce production plans, is able to achieve a Pareto improvement relative to most market outcomes. The function of this planner can be decentralized: It is possible to define objectives of the producer that aim at welfare maximization under the constraint of individual rationality. Therefore, it is possible to reconcile normative and positive criteria, but these criteria are very weak.

\section{Limit}

A classical result for complete markets is that as the number of producers becomes large, and thus each of them becomes small relative to the size of the economy, Cournot-Walras equilibria converge to competitive equilibria. In competitive equilibrium, producers behave as price takers and maximize profits with respect to a given state price vector $q \in \mathbb{R}^{|\boldsymbol{\Omega}|}$. Equilibrium asset prices are characterized by the condition $p=q \cdot \boldsymbol{A}$, and since markets are complete, the state price vector is unique and equal to the marginal cost vectors of all firms; i.e., $q=D \kappa^{k}\left[A^{k}\right] \forall k$. Competitive equilibria are desirable from a normative viewpoint as they result in a Pareto efficient allocation.

The purpose of this section is to analyze how the nature of this result changes when Cournot-Walras equilibria with incomplete markets are permitted. As in Gabszewicz and Vial (1972), the base economy $(U, e, \mathcal{A}, \Pi)$ is enlarged by means of replication: The $n$th replication is an economy populated by $n$ identical copies of each consumer $i$ and $n$ identical copies of each producer $k$. The focus is on the set of Cournot-Walras equilibria for the limit economy as $n \rightarrow \infty$. Four questions are addressed: Does the indeterminacy vanish in the limit? Are markets complete? Do producers behave as if they were price takers? Are limit equilibria efficient, at least in constrained sense?

To answer these questions, the example from Sect. 3 is replicated. In this example, all four questions are answered in the negative. It is sufficient to consider sequences of symmetric equilibria. Even though Cournot-Walras equilibria need not exist at all stages of replication in general, the example is sufficiently well behaved that nonexistence problems do not occur:

Claim 1 At each stage of replication n, there exists a Cournot-Walras equilibrium with symmetric strategies. 


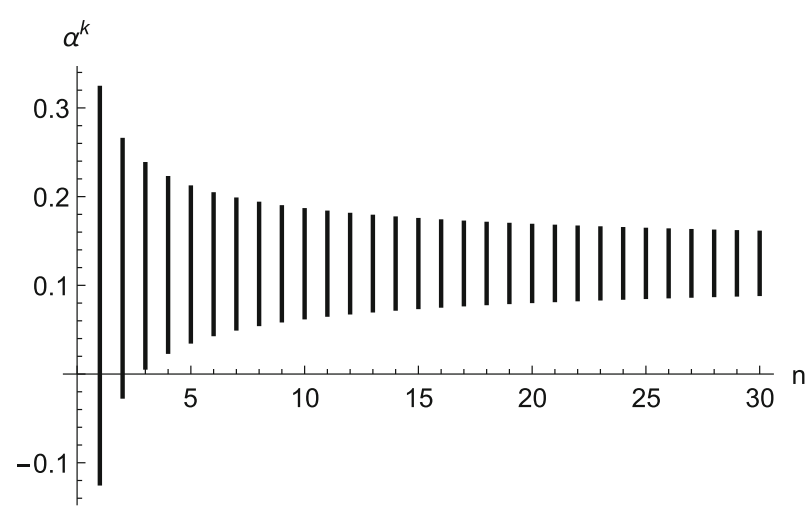

Fig. 4 Sets of symmetric equilibrium strategies for replications $n=1, \ldots, 30$

Claim 1 is easily proven by inspection of the market price function and its second-order derivatives. The details can be found in the appendix. The advantage of symmetric equilibria is that the market price at every stage $n$ can be written as a function $p_{n}^{k *}\left(\alpha^{k}, \alpha^{\neg k}\right)$ of two arguments: $\alpha^{k}$ is a scalar that represents the strategy of producer $k$, and $\alpha^{-k}$ is a scalar that represents the strategy played by each of the other $K n-1$ producers. As a consequence, the set of symmetric equilibria can at any stage be computed as in a two-player game. Figure 4 depicts this set for the first 30 replications. The equilibrium intervals are drawn along the vertical axis as the number of replications increases along the horizontal axis.

The base economy is populated by $I=2$ consumers and $K=2$ producers, and all symmetric strategy combinations $\alpha^{1}=\alpha^{2}$ in the interval [ $\left.-0.123,0.322\right]$ induce a Cournot-Walras equilibrium. In the next round of replication, there are $2 I=4$ consumers and $2 K=4$ producers. All four producers choose identical strategies $\alpha^{k}$, but now the set of equilibrium strategies is the smaller interval $[-0.022,0.266]$. In every further round of replication, two replicas of the original consumers and two replicas of the original producers are added, and the set of equilibrium strategies shrinks further. With each replication, the set of equilibrium strategy combinations becomes smaller, but the dimension of the set stays the same. This is a direct consequence of Theorem 1: The dimension of the equilibrium set is the sum of the degree of real indeterminacy $r(|\boldsymbol{\Omega}|-r)=1$ and the degree of financial indeterminacy $K n-r=2 n-$ 1. As the economy is replicated, more and more redundant assets are introduced, which increases the degree of financial indeterminacy while the degree of real indeterminacy remains unchanged. In order to understand why incomplete markets remain optimal for the individual producers, the payoff function of Producer 1 is studied as the economy is enlarged. Figure 5 depicts how the shape of this function changes progressively from $n=1$, over $n=2$, to $n=30$.

As shown in Fig. 2, the equilibrium strategies are fixed for all other producers. It is easy to see that the payoff function becomes flatter and flatter. Since production costs are zero in the example, this is a perfect reflection of the market price function of the first asset. As the output of a single producer becomes negligible relative to the size of the economy, his influence on prices diminishes provided the rank of the asset payoff 

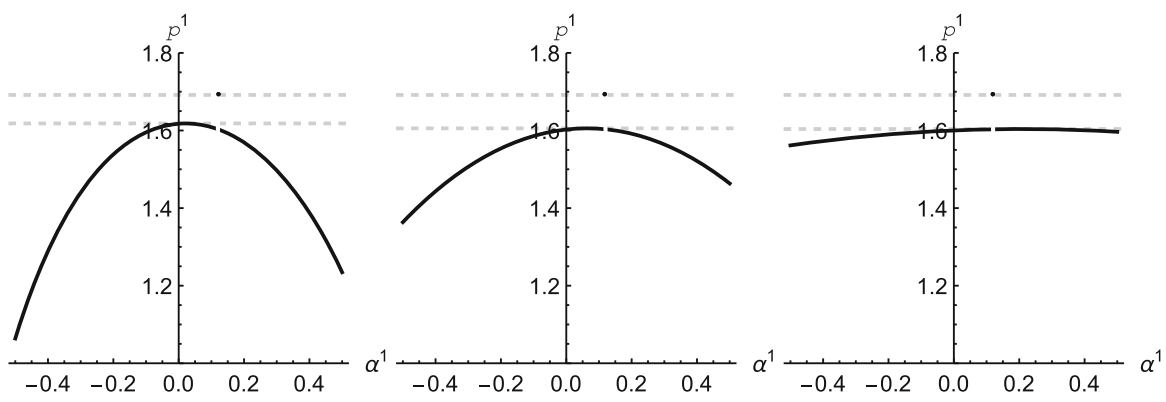

Fig. 5 Profit of Producer 1 as a function of $\alpha^{1}$ in replications $n=1, n=2$, and $n=30$. All other producers play $\alpha^{2}=\cdots=\alpha^{2 n}=0.12$

matrix does not change. The impact of such a rank change, however, remains large even if the producer becomes relatively small. It appears that $p_{n}^{k *}$ tends to a function linear in $\alpha^{k}$ except at the point of discontinuity as $n \rightarrow \infty$. This impression is correct:

Claim 2 There are functions $\beta, \gamma$, and $\delta$ such that

$$
\lim _{n \rightarrow \infty} p_{n}^{k *}\left(\alpha^{k}, \alpha^{\neg k}\right)=\left\{\begin{array}{ll}
\beta\left(\alpha^{\neg k}\right) & \text { if } \alpha^{k}=\alpha^{\neg k} \\
\gamma\left(\alpha^{\neg k}\right)+\delta\left(\alpha^{\natural}\right) \alpha^{k} & \text { otherwise }
\end{array} .\right.
$$

Claim 2 answers the questions whether the indeterminacy of Cournot-Walras equilibrium vanishes in the limit economy. All its symmetric equilibria can be found by solving the inequality $\gamma\left(\alpha^{k}\right)+\delta\left(\alpha^{k}\right) \alpha^{k} \leq \beta\left(\alpha^{k}\right)$ for $\alpha^{k}$. This inequality has a continuum of solutions, and thus the limit economy has a continuum of equilibria. All strategy combinations $\alpha^{k} \in[0.087,0.155] \forall k$ induce a Cournot-Walras equilibrium. This also answers the question whether limit equilibria involve complete markets: Not necessarily. Symmetric strategies result in 1-dimensional asset spans. Thus, the entire continuum consists of Cournot-Walras equilibria with incomplete markets.

The question whether producers behave as price takers is more delicate. Since incompleteness entails a multiplicity of state prices, there is no unambiguous notion of price-taking producers. An extension of price-taking behavior to incomplete market economies that is based on efficiency considerations is presented by Drèze (1974). Under his criterion, each producer $k$ takes as given a subjective state price vector $q^{k}$ defined as a weighted sum of consumers' marginal rates of substitution. In the present setting with quasilinear utility, it can be written as

$$
q^{k}=\sum_{i=1}^{I} \psi_{k}^{i} D u^{i}\left[c_{\mathbf{1}}^{i}\right]
$$

Each producer uses his subjective state price vector to estimate the market price of the asset he issues. If the concept of Cournot-Walras equilibrium is modified in such a way that producers maximize profits based on these price estimates, one speaks of Drèze equilibrium. 
Definition 5 A Drèze equilibrium for economy $(U, e, \mathcal{A}, \Pi)$ is a tuple $(\boldsymbol{A}, p, c, \psi)$ such that

$$
\boldsymbol{A}^{k} \in \underset{\boldsymbol{A}_{\#}^{k}}{\arg \max } \sum_{i=1}^{I} \psi_{k}^{i} D u^{i}\left[c_{\mathbf{1}}^{i}\right] \cdot \boldsymbol{A}_{\#}^{k}-\kappa^{k}\left(\boldsymbol{A}_{\#}^{k}\right) \quad \text { subject to } \quad \boldsymbol{A}_{\#}^{k} \in \mathcal{A}^{k} \quad \forall k,
$$

as well as $p=p^{*}(\boldsymbol{A}), c=c^{*}(\boldsymbol{A}, p), \psi \in \Psi^{*}(\boldsymbol{A}, p)$, and $\bar{\psi}=\mathbf{1}$.

If markets are complete at a Drèze equilibrium, all utility gradients are equalized and so are the subjective state prices of producers. Therefore, in this special case, the behavior of producers boils down to objective price-taking as in competitive equilibrium.

It is easy to check whether a Cournot-Walras equilibrium is a Drèze equilibrium, and such a comparison is also helpful to answer the efficiency question: A Cournot-Walras equilibrium is Pareto efficient if and only if it is a Drèze equilibrium with complete markets. When markets are incomplete, Pareto efficiency is typically not attainable, but then there is a connection to the weaker standard of constrained efficiency, originally introduced by Diamond (1967). This standard can be described by means of a social planner who can choose production plans, asset allocations, and date 0 consumption freely. Constrained efficiency is attained if such a planner cannot achieve a Pareto improvement. The most important property in the present context is: If a CournotWalras equilibrium is constrained efficient, then it is a Drèze equilibrium.

It can be verified that only one of the symmetric Cournot-Walras equilibria satisfies the condition $p=q^{k} \cdot \boldsymbol{A}$. It is the equilibrium at $\alpha^{k}=0.087 \forall k$, which maximizes welfare within the set of symmetric strategy combinations. This is the only Drèze equilibrium within the continuum, and thus the only candidate for constrained efficiency. Nevertheless, the equilibrium has rather undesirable properties: A welfare improvement is possible if a significant share of the producers, for example half of them, changes their production plan slightly such that markets are complete. Thus, the Drèze equilibrium at hand fails to be constrained efficient. ${ }^{9}$ As a consequence, all sequences of symmetric Cournot-Walras equilibria have a constrained inefficient limit.

The behavior of producers in the limit economy exhibits some similarity with pricetaking behavior. Restricted to each rank $r$, producers face a linear market price function. Therefore, they evaluate production plans as if state prices were fixed and independent of production choices. In fact, the output quantity of each producer is negligible, such that an individual change would not affect prices. On the other hand, each producer can still affect market prices by offering an asset that increases the dimension of the asset span. Therefore, some form of market power survives in the limit when producers are small. Moreover, each producer fully takes into account this market power. Markets are not incomplete out of neglect or out of perceived competitive behavior; they are incomplete because each producer, in full awareness of his market power, decides not to enlarge the asset span.

\footnotetext{
9 For a detailed discussion of constrained inefficient Drèze equilibria when assets are redundant, the reader is referred to Zierhut (2019).
} 


\section{Conclusion}

Endogenous asset structure choice in Cournot-Walras equilibrium results in a discontinuous game. If producers choose to issue redundant assets, the equilibrium lies right at a discontinuity, and such equilibria are never isolated: The set of regular equilibria is a continuum whose dimension grows with the number of redundant assets. Therefore, the set of market outcomes that are individually rational for producers grows larger as markets become more incomplete.

The incentive to create an incomplete asset structure does not disappear when the number of producers grows large. Even in the limit economy, where the output of each producer is negligible relative to the size of the market, there is still market power in the ability to offer novel assets. However, producers may find it profitable not to use this power, and market incompleteness is preserved in equilibrium. A multiplicity of equilibria with different asset spans can be found in economics with large producers and in economies with small producers alike.

From a positive viewpoint, all equilibria are on par. Each of them is perfectly consistent with the self-interest of producers. However, from a normative viewpoint, not all equilibria are equally desirable. Different equilibria can be ranked in terms of welfare. Therefore, even a power-oriented setting with producers who behave strategically leaves sufficient room for normative theory. Welfare considerations are not obsolete but may be used to guide production decisions.

The implications for the theory of the firm are self-evident: Even if the firm takes its own market power fully into account, it can be equipped with an objective that is both individually rational for its owners and socially desirable in a weak sense. In a sole proprietorship economy, individual rationality is well-defined since there is only one type of owner. Other forms of ownership require a broader concept of rationality, and welfare maximization among the group of owners may serve as the basis. Developing objectives of the firm that resolve the indeterminacy in a socially desirable way is a promising direction for future research.

\section{Acknowledgements Open Access funding provided by Projekt DEAL.}

Open Access This article is licensed under a Creative Commons Attribution 4.0 International License, which permits use, sharing, adaptation, distribution and reproduction in any medium or format, as long as you give appropriate credit to the original author(s) and the source, provide a link to the Creative Commons licence, and indicate if changes were made. The images or other third party material in this article are included in the article's Creative Commons licence, unless indicated otherwise in a credit line to the material. If material is not included in the article's Creative Commons licence and your intended use is not permitted by statutory regulation or exceeds the permitted use, you will need to obtain permission directly from the copyright holder. To view a copy of this licence, visit http://creativecommons.org/licenses/by/4.0/.

\section{Appendix}

The appendix is composed of two parts. The first part introduces the reader to vector bundles and presents results in differential topology that are preliminary to proving the main theorem of this paper. Its centerpiece is the derivation of an implicit function theorem for bundle mappings. This part is self-contained and may be of independent 
interest. The second part connects those general results with the economic model of this paper. It contains detailed proofs of Theorem 1, its corollaries, and all propositions and claims.

\section{A.1 Preliminaries}

The preimage theorem (see Villanacci et al. (2002), p. 125, Theorem 49) is a useful tool for describing the solution set to a system of differentiable equations. If the equation system is represented by $f(x)=z$, in which $f: X \rightarrow Z$ is a $C^{s}$ function between two $C^{s}$ manifolds $X, Z$, then the preimage theorem states that $f^{-1}(z)$ is a $C^{s}$ manifold of dimension equal to $\operatorname{codim}(Z)$ provided that $f$ is transverse to $\{z\}$. A function $f$ is transverse to a manifold $Y$, written $f \pitchfork Y$, if its differential $d f[x]$ is surjective at all $x \in f^{-1}(Y)$. The below example illustrates that transversality can be a restrictive condition. Let $\mathcal{M}_{r}(m, n)$ be the set of $m \times n$ matrices with rank $r$. The following lemma recollects a well-known property of this set. A detailed proof can be found in Govaerts (2014), Sect. 4.

Lemma $\mathrm{A} 1$ The set $\mathcal{M}_{r}(m, n)$ of $m \times n$ matrices with rank $r$ is a smooth manifold of dimension $r(m+n)-r^{2}$.

Example Consider the smooth function $g: \mathcal{M}_{2}(3,2) \times \mathbb{R}^{3} \rightarrow \mathbb{R}^{3}$ defined as $g(\boldsymbol{M}, x)=\boldsymbol{M} \cdot\left(\boldsymbol{M}^{\top} \cdot \boldsymbol{M}\right)^{-1} \cdot \boldsymbol{M}^{\top} \cdot x$. By Lemma A1, $g$ is a function between smooth manifolds. It projects each vector $x$ onto the linear subspace spanned by the columns of $\boldsymbol{M}$. It is clear that the preimage $g^{-1}(0)$ must be a smooth manifold, yet the preimage theorem cannot be applied to determine its dimension because $g \not\{\{0\}$.

The problem in the example can be overcome if $g$ is recast as a bundle mapping. A bundle mapping is a function with additional structure between vector bundles. A detailed treatment of vector bundles can be found in Hirsch (1994), Chapter 4. Here, the most important aspects are summarized briefly: A $C^{s}$ vector bundle $V$ is constructed by attaching a vector space to each point of a $C^{s}$ base manifold $\mathcal{B}$. The vector space attached to point $b \in \mathcal{B}$ is called fiber of $V$ at $b$, written $V[b]$. On open subsets $\mathcal{O} \in \mathcal{B}$, the fiber varies in a $C^{s}$ manner: The entire vector bundle $V$ is described by $C^{s}$ homeomorphisms, called vector bundle charts, of the form $f: \mathcal{N} \rightarrow \mathcal{O} \times \mathbb{R}^{n}$ in which $\mathcal{N}$ is an open subset of $V$. Therefore, each vector bundle is everywhere locally homeomorphic to a product of an open subset of the base manifold and some Euclidean space (of dimension $n=\operatorname{dim}(V[b])$ ). As a consequence, every $C^{s}$ vector bundle is a $C^{s}$ manifold itself. The converse is true as well: Every $C^{s}$ manifold can be viewed as a $C^{s}$ vector bundle with zero-dimensional fibers.

Let $V_{X}, V_{Y}$ be two $C^{s}$ vector bundles over base manifolds $\mathcal{B}_{X}, \mathcal{B}_{Y}$. A $C^{s}$ bundle mapping is a $C^{s}$ function $h: V_{X} \rightarrow V_{Y}$ that has the form $h(b, x)=(\varphi(b), h[b](x))$, in which $h[b]: V_{X}[b] \rightarrow V_{Y}[\varphi(b)]$ is a $C^{s}$ function between fibers, and $\varphi: \mathcal{B}_{X} \rightarrow \mathcal{B}_{Y}$ is a $C^{s}$ function between the base manifolds. In this case, $h$ is said to cover $\varphi$. A bundle mapping is therefore a way of modeling families of functions $h[b]$ whose domains and ranges depend in a $C^{s}$ manner on the parameter $b$. One way of defining $C^{s}$ vector bundles is by means of a $C^{s}$ function $F: \mathcal{B} \rightarrow \mathcal{G}_{r}(m)$ from the base manifold to the set of all $r$-dimensional linear subspaces of $\mathbb{R}^{m}$. The properties of this set $\mathcal{G}_{r}(m)$, 
which is often times referred to as Grassmannian, are summarized in the following lemma. For a proof, the reader is referred to Duffie and Shafer (1985), Sect. 4.

Lemma A2 The set $\mathcal{G}_{r}(m)$ of $r$-dimensional linear subspaces of $\mathbb{R}^{m}$ is a compact, smooth manifold of dimension $r(m-r)$.

The two manifolds $\mathcal{M}_{r}(m, n)$ and $\mathcal{G}_{r}(m)$ are closely related: Every element $\boldsymbol{M}$ of the former is associated with an element $L$ of the latter through its image $L=\operatorname{Im}(\boldsymbol{M})$. The following lemma makes this relation more precise and can be stated without proof.

Lemma A3 There is a smooth submersion $\varphi: \mathcal{M}_{r}(m, n) \rightarrow \mathcal{G}_{r}(m)$ with $\operatorname{Ker}(d \varphi[\boldsymbol{M}])$ $=\operatorname{Im}(\boldsymbol{M})^{n}$.

Consider the disjoint union

$$
\mathcal{V}(\mathcal{B}, F)=\bigcup_{b \in \mathcal{B}}\{b\} \times F(b),
$$

which is a vector bundle by the following lemma.

Lemma A4 If $\mathcal{B}$ is a $C^{s}$ manifold and $F \in C^{s}\left(\mathcal{B}, \mathcal{G}_{r}(m)\right)$, then $\mathcal{V}(\mathcal{B}, F)$ is a $C^{s}$ vector bundle.

Proof For every linear subspace $L \in \mathcal{G}_{r}(m)$, there is a smooth homeomorphism $h[L]$ : $L \rightarrow \mathbb{R}^{r}$. In particular, there is an open neighborhood $\mathcal{N} \subset \mathcal{G}_{r}(m)$ of $L$, and there is a smooth vector bundle $\mathcal{N}^{2}=\{(L, L) \mid L \in \mathcal{N}\}$, such that $h: \mathcal{N}^{2} \rightarrow \mathcal{N} \times \mathbb{R}^{r}$ is a smooth bundle mapping over the identity. The set $\mathcal{O}=F^{-1}(\mathcal{N})$ is open by continuity of $F$. Define a function $g^{-1}: \mathcal{O} \times \mathbb{R}^{r} \rightarrow \mathcal{V}(\mathcal{B}, F)$ as $g^{-1}(b, x)=(b, h(F(b), x))$. Note that $g^{-1}$ is of class $C^{s}$ because $F$ is and all other functions are smooth. Its Jacobian in local coordinates is

$$
D g^{-1}[b, x]=\left(\begin{array}{cc}
\boldsymbol{I} & \mathbf{0} \\
D_{L} h[F(b), x] \cdot D F[b] & D_{x} h[F(b), x]
\end{array}\right),
$$

which has full rank since $D_{x} h[F(b), x]$ is invertible because $h[F(b)]$ is a homeomorphism. By the inverse function theorem, $\left.g\right|_{\mathcal{P}}$ is a $C^{s}$ homeomorphism for $\mathcal{P}=g^{-1}\left(\mathcal{O} \times \mathbb{R}^{r}\right)$. Thus, $\left.g\right|_{\mathcal{P}}$ is a vector bundle chart of $\mathcal{V}(\mathcal{B}, F)$.

It should be noted that $V$ can be equipped with a $C^{s}$ homeomorphism $\sigma_{0}: \mathcal{B} \rightarrow V$ that satisfies $\sigma_{0}(b)=f^{-1}(b, 0) \forall b \in \mathcal{O}$ for any vector bundle chart $f: \mathcal{N} \rightarrow \mathcal{O} \times \mathbb{R}^{n}$. The image $\sigma_{0}(\mathcal{B})$ is an embedded $C^{s}$ submanifold called the zero section of $V .{ }^{10}$ It is easy to check whether a bundle mapping is transverse to the zero section of its range:

Lemma A5 Let $f: V_{X} \rightarrow V_{Y}$ be a $C^{s}$ bundle mapping that covers a submersion. If $d_{x} f[b, x]$ is surjective for all $(b, x) \in f^{-1}\left(\sigma_{0}\left(\mathcal{B}_{Y}\right)\right)$, then $f \pitchfork \sigma_{0}\left(\mathcal{B}_{X}\right)$.

\footnotetext{
${ }^{10}$ It should be noted that other authors prefer to call the function $\sigma_{0}$ itself the zero section.
} 
Proof Denote by $\varphi$ the $C^{s}$ submersion and by $n$ the dimension of the base manifolds. As $\varphi$ is a submersion, $d \varphi[b]$ is surjective and the Jacobian of $f$ in local coordinates

$$
D f[b, x]=\left(\begin{array}{cc}
D \varphi[b] & \mathbf{0} \\
D_{b} f[b, x] & D_{x} f[b, x]
\end{array}\right)
$$

has full rank whenever $d_{x} f[b, x]$ is surjective.

Now, it is time to nest the function $g$ from the example within a new function $\tilde{g}: \mathcal{V}\left(\mathcal{M}_{2}(3,2), \boldsymbol{M} \mapsto \mathbb{R}^{3}\right) \rightarrow \mathcal{V}\left(\mathcal{M}_{2}(3,2), \boldsymbol{M} \mapsto \operatorname{Im}(\boldsymbol{M})\right)$ of the form $\tilde{g}(\boldsymbol{M}, x)=(\boldsymbol{M}, g(\boldsymbol{M}, x))$. By Lemma A4, domain and range of $\tilde{g}$ are smooth vector bundles. In particular, since the range has a product structure globally, its zero section is simply $\mathcal{M}_{2}(3,2) \times\{0\}$. Moreover, $\tilde{g}$ is a smooth bundle mapping covering the identity. Since $\operatorname{Im}\left(d_{x} \tilde{g}[\boldsymbol{M}]\right)=\operatorname{Im}(\boldsymbol{M})$ by construction, the differential is always surjective and Lemma A5 ensures that $\tilde{g} \pitchfork \mathcal{M}_{2}(3,2) \times\{0\}$. Furthermore, $g^{-1}(0)=\tilde{g}^{-1}\left(\mathcal{M}_{2}(3,2) \times\{0\}\right)$ by construction, and therefore, the preimage theorem applied to $\tilde{g}$ establishes that $g^{-1}(0)$ is a smooth manifold of dimension

$$
\begin{aligned}
\operatorname{dim}\left(g^{-1}(0)\right)= & \operatorname{dim}\left(\mathcal{V}\left(\mathcal{M}_{2}(3,2), \boldsymbol{M} \mapsto \mathbb{R}^{3}\right)\right)-\operatorname{dim}\left(\mathcal{V}\left(\mathcal{M}_{2}(3,2), \boldsymbol{M} \mapsto \operatorname{Im}(\boldsymbol{M})\right)\right) \\
& +\operatorname{dim}\left(\mathcal{M}_{2}(3,2) \times\{0\}\right) \\
= & \operatorname{dim}\left(\mathbb{R}^{3}\right)-\operatorname{dim}(\operatorname{Im}(\boldsymbol{M}))+\operatorname{dim}\left(\mathcal{M}_{2}(3,2)\right) \\
= & 3-2+\operatorname{dim}\left(\mathcal{M}_{2}(3,2)\right),
\end{aligned}
$$

and thus by Lemma A1, $\operatorname{dim}\left(g^{-1}(0)\right)=7$. Therefore, the additional structure of a bundle mapping enables the application of the preimage theorem in cases in which transversality in a narrow sense is not satisfied.

The following two lemmata show that new vector bundles can be formed by means of splitting and joining.

Lemma A6 Let $V_{X \times Y}$ be a $C^{s}$ vector bundle over a manifold $\mathcal{B}$. Then, there exist $C^{s}$ vector bundles $V_{X}$ and $V_{Y}$ over $\mathcal{B}$ whose fibers satisfy $V_{X}[b] \times V_{Y}[b]=V_{X \times Y}[b]$.

Proof Every vector bundle chart $f: \mathcal{N} \rightarrow \mathcal{O} \times \mathbb{R}^{m} \times \mathbb{R}^{n}$ is a $C^{s}$ bundle mapping covering $\sigma_{0}^{-1}$. As $V_{X \times Y}[b]$ is a vector space, the local coordinates can always be chosen in such a way that the Jacobian of the inverse $f^{-1}$ has all off-diagonal blocks equal to the zero matrix:

$$
D f^{-1}[b, x, y]=\left(\begin{array}{ccc}
D \sigma_{0}[b] & \mathbf{0} & \mathbf{0} \\
\mathbf{0} & D_{x} f^{-1}[b, x, y] & \mathbf{0} \\
\mathbf{0} & \mathbf{0} & D_{y} f^{-1}[b, x, y]
\end{array}\right) .
$$

Define $C^{s}$ functions $g^{-1}: \mathcal{O} \times \mathbb{R}^{m} \rightarrow \mathcal{N}_{X}$ with $\mathcal{N}_{X}=f^{-1}\left(\mathcal{O} \times \mathbb{R}^{m} \times\{0\}\right)$ and $h^{-1}: \mathcal{O} \times \mathbb{R}^{n} \rightarrow \mathcal{N}_{Y}$ with $\mathcal{N}_{Y}=f^{-1}\left(\mathcal{O} \times\{0\} \times \mathbb{R}^{n}\right)$ as follows: $g^{-1}(b, x)=$ $f^{-1}(b, x, 0)$ and $h^{-1}(b, y)=f^{-1}(b, 0, y)$. Note that $d g^{-1}[b, x]$ and $d h^{-1}[b, y]$ are 
both bijective; otherwise, $D f^{-1}[b, x, y]$ in (10) could not have full rank, which would contradict that $f$ is a vector bundle chart. Thus, by the inverse function theorem for $C^{s}$ manifolds, $g$ and $h$ are homeomorphisms. They serve as vector bundle charts for $V_{X}$ and $V_{Y}$, respectively.

Lemma A7 Let $V_{X}, V_{Y}$ be $C^{s}$ vector bundles, and let $f: V_{X} \rightarrow V_{Y}$ be a $C^{s}$ bundle mapping covering $\varphi$. If $\varphi$ is a submersion, there is exists a $C^{s}$ vector bundle $V_{X \times Y}$ such that $V_{X \times Y}[b]=V_{X}[b] \times V_{Y}[\varphi(b)]$.

Proof Let $\mathcal{B}_{X}$ and $\mathcal{B}_{Y}$ be the base manifolds of $V_{X}$ and $V_{Y}$, respectively. Since $\varphi$ is a submersion, it translates any open cover of $\mathcal{B}_{X}$ to an open cover of $\mathcal{B}_{Y}$. In particular, for any pair of vector bundle charts $g: \mathcal{N}_{X} \rightarrow \mathcal{O}_{X} \times \mathbb{R}^{m}$ of $V_{X}$ and $h: \mathcal{N}_{Y} \rightarrow \mathcal{O}_{Y} \times \mathbb{R}^{n}$ of $V_{Y}$ such that $\varphi\left(\mathcal{O}_{X}\right) \cap \mathcal{O}_{Y}$ is nonempty, one can define a set $\mathcal{P}=\varphi^{-1}\left(\varphi\left(\mathcal{O}_{X}\right) \cap \mathcal{O}_{Y}\right)$ a function $\ell^{-1}: \mathcal{P} \times \mathbb{R}^{m} \times \mathbb{R}^{n}$ as $\ell^{-1}(b, x, y)=\left(g^{-1}(b, x), \operatorname{pr}_{Y}\left(h^{-1}(\varphi(b), y)\right)\right)$. Then, $\ell^{-1}$ is a $C^{s}$ homeomorphism because $g^{-1}$ and $h^{-1}$ are, and $\ell$ is a vector bundle chart of $V_{X \times Y}$.

Finally, a vector bundle version of the familiar implicit function theorem is derived.

Theorem A1 Let $V_{X \times Y}, V_{Z}$ be $C^{s}$ vector bundles, and let $f: V_{X \times Y} \rightarrow V_{Z}$ be a $C^{s}$ bundle mapping that covers a homeomorphism. If $f[b](x, y)=z$ and $d_{x} f[b, x, y]$ is bijective, then there are open neighborhoods $\mathcal{N}$ of $(b, x)$ in $V_{X}$ and $\mathcal{O}$ of $(b, y)$ in $V_{Y}$, and a unique $C^{s}$ bundle mapping $g: \mathcal{O} \rightarrow \mathcal{N}$ that satisfies $\left.f[b](g[b](y), y)\right)=z$ for all $(b, y) \in \mathcal{O}$.

Proof Denote by $\varphi$ the $C^{s}$ homeomorphism. By Lemmata A6 and A7, it is possible to split $V_{X \times Y}$ in two and glue one part to $V_{Z}$. This results in a $C^{s}$ vector bundle $V_{Z \times Y}$. Now, define the $C^{s}$ function $h: V_{X \times Y} \rightarrow V_{Z \times Y}$ as $h(b, x, y)=(f(b, x, y), y)$. Note that bijectivity of $d_{x} f[b, x, y]$ implies $\operatorname{dim}(X)=\operatorname{dim}(Z)$, and thus, range and domain of $h$ are of the same dimension. The Jacobian in local coordinates

$$
D_{b, x} h[b, x, y]=\left(\begin{array}{ccc}
D \varphi[b] & \mathbf{0} & \mathbf{0} \\
D_{b} f[b, x, y] & D_{x} f[b, x, y] & D_{y} f[b, x, y] \\
\mathbf{0} & \mathbf{0} & \boldsymbol{I}
\end{array}\right)
$$

has full rank since both $D_{x} f[b, x, y]$ and $D \varphi[b]$ have full rank, the latter because $\varphi$ is a homeomorphism. As a consequence, the inverse function theorem for $C^{s}$ manifolds (see Villanacci et al. (2002), p. 67, Theorem 48) guarantees the existence of a $C^{s}$ inverse $h^{-1}$. Note that $h$ can be written as $h(b, x, y)=(\varphi(b), f[b](x, y), y)$, and thus by the positions of the zeroes in (11), it is clear that $h^{-1}$ is of the form $h^{-1}\left(b^{\prime}, x^{\prime}, y^{\prime}\right)=\left(\varphi^{-1}\left(b^{\prime}\right), \ell\left[b^{\prime}\right]\left(x^{\prime}, y^{\prime}\right), y^{\prime}\right)$ for some $\ell: V_{Z \times Y} \rightarrow V_{X}$ that satisfies $f\left[\varphi^{-1}\left(b^{\prime}\right)\right]\left(\ell\left[b^{\prime}\right]\left(x^{\prime}, y^{\prime}\right), y^{\prime}\right)=x^{\prime}$; otherwise, $h \circ h^{-1} \neq \operatorname{id}_{V_{X \times Y}}$. As a consequence, $g[b](y)=\ell[\varphi(b)](z, y)$ is the desired bundle mapping that solves $f[b](g[b](y), y))=z$. 


\section{A.2 Proofs}

Since only regular equilibria are studied, the topological boundaries bdry $\left(\mathcal{A}^{k}\right)$ of production sets can be ignored. Under Assumption 3, the set $\tilde{\mathcal{A}}^{k}=\mathcal{A}^{k} \backslash \operatorname{bdry}\left(\mathcal{A}^{k}\right)$ is nonempty-open and therefore a smooth $|\boldsymbol{\Omega}|$-dimensional manifold. As a consequence, $\tilde{\mathcal{A}}=\chi_{k=1}^{K} \tilde{\mathcal{A}}^{k}$ is a smooth $K|\boldsymbol{\Omega}|$-dimensional manifold. It overlaps with the set $\mathcal{M}_{r}(|\boldsymbol{\Omega}|, K)$ of $|\boldsymbol{\Omega}| \times K$ matrices with rank $r$, which is a smooth manifold itself by Lemma A1. Since $T \tilde{\mathcal{A}}[\boldsymbol{A}]=\mathbb{R}^{K|\boldsymbol{\Omega}|}$ for any $\boldsymbol{A} \in \tilde{\mathcal{A}}$, the intersection with any other manifold is trivially transverse; in particular, $\tilde{\mathcal{A}} \pitchfork \mathcal{M}_{r}$. As a consequence, the intersection $\tilde{\mathcal{A}}_{r}=\tilde{\mathcal{A}} \cap \mathcal{M}_{r}$ is another smooth manifold of dimension $r(|\boldsymbol{\Omega}|+K)-r^{2}$. Consider the set

$$
\tilde{\mathcal{X}}_{r}=\mathcal{V}\left(\tilde{\mathcal{A}}_{r}, \boldsymbol{A} \mapsto \operatorname{Im}\left(\boldsymbol{A}^{\top}\right)\right)
$$

which is a smooth vector bundle over $\tilde{\mathcal{A}}_{r}$ by Lemma A4. By construction, if $(\boldsymbol{A}, p) \in$ $\mathcal{X}_{r}$ (as defined in Eq. (6)) and $\boldsymbol{A} \notin \operatorname{bdry}(\mathcal{A})$, then $(\boldsymbol{A}, p) \in \tilde{\mathcal{X}}_{r}$. The objective is to study the set $\Xi_{r}^{* *}$ of strongly regular equilibria with rank $r$. The following lemma establishes that the dimension of $\Xi_{r}^{* *}$ is equal to the dimension of the tractable set $\hat{\Xi}_{r}^{*}$ :

Lemma A8 For any $r \in \mathbb{N}$, the set of strongly regular Cournot-Walras equilibria $\Xi_{r}^{* *}$ is open in the set of regular restricted equilibria $\hat{\Xi}_{r}^{*}$.

Proof By Definition 3, the best-reply correspondence $A^{*}$ is single-valued at any $(\boldsymbol{A}, p, c, \psi) \in \boldsymbol{\Xi}_{r}^{*}$. This implies that for each producer $k$,

$$
\Pi^{k *}\left(\boldsymbol{A}^{k}, \boldsymbol{A}^{\neg k}\right)>\max _{\boldsymbol{A}_{\#}^{k}} \Pi^{k *}\left(\boldsymbol{A}_{\#}^{k}, \boldsymbol{A}^{\neg k}\right) \text { subject to } \boldsymbol{A}_{\#}^{k} \in \mathcal{A}^{k} \backslash \operatorname{Im}(\boldsymbol{A}) .
$$

If $(\boldsymbol{A}, p, c, \psi) \in \hat{\boldsymbol{\Xi}}_{r}^{*}$, then also for each $k$,

$$
\Pi^{k *}\left(\boldsymbol{A}^{k}, \boldsymbol{A}^{\neg k}\right)=\max _{\boldsymbol{A}_{\#}^{k}} \Pi^{k *}\left(\boldsymbol{A}_{\#}^{k}, \boldsymbol{A}^{\neg k}\right) \text { subject to } \boldsymbol{A}_{\#}^{k} \in \mathcal{A}^{k} \cap \operatorname{Im}(\boldsymbol{A}) .
$$

Note that Definition 3 implies that $A^{*}$ is continuous at the equilibrium, which implies that the maximum in (13) is locally continuous in the first argument $\boldsymbol{A}_{\#}^{k}$. Thus, strict inequality (12) holds in some neighborhood $\mathcal{N} \subset \hat{\Xi}_{r}^{*}$ of the original equilibrium, and thus, $\xi \in \mathcal{N}$ implies $\xi \in \Xi_{r}^{* *}$.

The advantage of working with the restricted game is that the dimension of the asset span is held constant, and therefore, the market price function is continuous. Still, the best-reply correspondence $\hat{A}^{*}: \mathcal{A}_{r} \rightrightarrows \mathcal{A}_{r}$ may behave erratically at discontinuities of the correspondence $\hat{\mathcal{A}}_{r}$, which are easy to see when the correspondence is written in the form

$$
\hat{\mathcal{A}}_{r}^{k}\left(\boldsymbol{A}_{\#}\right)=\left\{\begin{array}{ll}
\mathcal{A}^{k} \cap \operatorname{Im}\left(\boldsymbol{A}_{\#}^{\neg k}\right) & \text { if } \operatorname{rank}\left(\boldsymbol{A}_{\#}^{\neg k}\right)=r \\
\mathcal{A}^{k} \backslash \operatorname{Im}\left(\boldsymbol{A}_{\#}^{-k}\right) & \text { if } \operatorname{rank}\left(\boldsymbol{A}_{\#}^{-k}\right)=r-1
\end{array} .\right.
$$


However, regular equilibria never occur at such discontinuities. Therefore, by Eq. (14) the choice set $\hat{\mathcal{A}}_{r}^{k}(\boldsymbol{A})$ of any producer $k$ at a regular equilibrium is contained in the linear subspace $L=\operatorname{Im}(\boldsymbol{A})$. Since the set of linear subspaces is a smooth manifold by Lemma A2, even the discontinuous correspondence $\hat{\mathcal{A}}_{r}$ can be equipped with a differentiable structure in a neighborhood of a regular equilibrium. For fixed $e \in \mathbb{E}$, all regular restricted equilibria are contained in the set

$$
\mathcal{Y}_{r}=\left\{\begin{array}{l|l}
(\boldsymbol{A}, p, c, \psi) \in \tilde{\mathcal{A}}_{r} \times \mathcal{R} & \begin{array}{c}
c^{i}-e^{i}=\left(\begin{array}{c}
-p \\
\boldsymbol{A}
\end{array}\right) \cdot \psi^{i} \forall i \\
p \\
\bar{\psi}=\operatorname{Im}\left(\boldsymbol{A}^{\top}\right) \\
\bar{\psi}=\mathbf{1}
\end{array}
\end{array}\right\},
$$

in which $\mathcal{R}=\mathbb{R}^{K} \times \mathbb{R}^{I(|\boldsymbol{\Omega}|+1)} \times \mathbb{R}^{I K}$ is the Euclidean space that contains all possible market outcomes $(p, c, \psi)$. Since the constraints in (15) are linear, $\mathcal{Y}_{r}$ is a vector bundle over $\tilde{\mathcal{A}}_{r}$. In the next step, a continuous selection from the demand correspondence shall be constructed. For this purpose, restricted equilibria are translated to restricted quasi-equilibria, which are free of financial indeterminacy. The linear subspace $L$ that contains the choice sets of all producers is made explicit in its definition:

Definition 6 A restricted quasi-equilibrium for economy $(U, e, \mathcal{A}, \Pi)$ is a tuple $(L, \boldsymbol{A}, p, c, \psi) \in \mathcal{G}_{r}(|\boldsymbol{\Omega}|) \times \mathcal{A}_{r} \times \mathbb{R}_{+}^{K} \times \mathcal{C} \times \mathbb{R}^{I K}$ such that $L=\operatorname{Im}(\boldsymbol{A}), \boldsymbol{A} \in \hat{A}^{*}(\boldsymbol{A})$, $p=p^{*}(\boldsymbol{A}), c=c^{*}(\boldsymbol{A}, p), \psi \in \Psi^{*}(\boldsymbol{A}, p) \cap \operatorname{Im}\left(\boldsymbol{A}^{\top}\right)^{I}$, and $\bar{\psi}-\mathbf{1} \in \operatorname{Ker}(\boldsymbol{A})$.

The main difference between Definitions 2 and 6 is in their degrees of freedom for portfolio choices. If there are redundant assets, the demand correspondence $\Psi^{*}$ involves a continuum of portfolios with identical payoffs. At a restricted quasiequilibrium, the choice of each consumer is confined to one single point of this continuum. As such confinement may preclude market clearing, the market clearing condition is weakened. While the original market clearing condition $\bar{\psi}-\mathbf{1} \in\{0\}$ is a $K$-dimensional restriction, the weaker clearing condition $\bar{\psi}-\mathbf{1} \in \operatorname{Ker}(\boldsymbol{A})$ is only a $(K-r)$-dimensional restriction. The set of restricted quasi-equilibria with rank $r$ is contained in the following set:

$$
\mathcal{Z}_{r}=\left\{(L, \boldsymbol{A}, p, c, \psi) \in \mathcal{G}_{r}(|\boldsymbol{\Omega}|) \times \tilde{\mathcal{A}}_{r} \times \mathcal{R} \mid \begin{array}{c}
c^{i}-e^{i}=\left(\begin{array}{c}
-p \\
\boldsymbol{A}
\end{array}\right) \cdot \psi^{i} \forall i \\
A \in L^{K} \\
p \in \operatorname{Im}\left(\boldsymbol{A}^{\top}\right) \\
\psi \in \operatorname{Im}\left(\boldsymbol{A}^{\top}\right)^{I}
\end{array}\right\} .
$$

Note that $\mathcal{Z}_{r}$ is a vector bundle over the Grassmannian $\mathcal{G}_{r}(|\boldsymbol{\Omega}|)$. Even though such restricted quasi-equilibria do not fulfill the market clearing condition of a restricted equilibrium, it is always possible to translate a regular equilibrium to a quasi-equilibrium and vice versa:

Lemma A9 For any $r \in \mathbb{N}$, there is a smooth submersion $\pi: \mathcal{Y}_{r} \rightarrow \mathcal{Z}_{r}$ having the following properties: 
(i) If $(\boldsymbol{A}, p, c, \psi)$ is a regular restricted equilibrium, then $\xi=\pi(\boldsymbol{A}, p, c, \psi)$ is a restricted quasi-equilibrium.

(ii) If $(L, \boldsymbol{A}, p, c, \psi)$ is a restricted quasi-equilibrium, then $\xi \in \pi^{-1}(L, \boldsymbol{A}, p, c, \psi)$ is a regular restricted equilibrium.

(iii) If $\xi$ is a restricted quasi-equilibrium, then $\operatorname{dim}\left(\pi^{-1}(\xi)\right)=(I-1)(K-r)$.

Proof Consider the bundle mapping $v: \tilde{A}_{r} \times \mathbb{R}^{K} \rightarrow \mathcal{V}\left(\tilde{A}_{r}, \boldsymbol{A} \mapsto \operatorname{Im}\left(\boldsymbol{A}^{\top}\right)\right)$ defined as

$$
v\left(\boldsymbol{A}, \psi^{i}\right)=\boldsymbol{A}^{\top} \cdot\left(\boldsymbol{A} \cdot \boldsymbol{A}^{\top}\right)^{-1} \cdot \boldsymbol{A} \cdot \psi^{i}
$$

which projects portfolio vectors onto the linear subspace spanned by the rows of $\boldsymbol{A}$. As a consequence, $\pi(\boldsymbol{A}, p, c, \psi)=\left(\varphi(\boldsymbol{A}), \boldsymbol{A}, p, c,\left(\nu[\boldsymbol{A}]\left(\psi^{i}\right)\right)_{i=1}^{I}\right)$ is a submersion because $\varphi$ is a submersion by Lemma A3. The three properties are proven separately:

Property $(i)$ By construction, $v[\boldsymbol{A}]\left(\psi^{i}\right) \in \operatorname{Im}\left(\boldsymbol{A}^{\top}\right)$ and $v[\boldsymbol{A}]\left(\psi^{i}\right)-\psi^{i} \in \operatorname{Ker}(\boldsymbol{A})$. As a consequence, $v[\boldsymbol{A}]\left(\psi^{i}\right) \in \Psi^{i *}(\boldsymbol{A}, p)$ because

$$
c^{i}=\boldsymbol{A} \cdot \psi^{i}=\boldsymbol{A} \cdot\left(\psi^{i}+\Delta \psi^{i}\right) \forall \Delta \psi^{i} \in \operatorname{Ker}(\boldsymbol{A}) .
$$

This is true for each consumer $i$, which results in the portfolio optimality condition from Definition 6. By the same logic, the weakened market clearing condition must hold: If $\bar{\psi}=\mathbf{1}$, then $v[\boldsymbol{A}](\bar{\psi})-\mathbf{1} \in \operatorname{Ker}(\boldsymbol{A})$.

Property (ii) By construction, $\psi^{i}+\Delta \psi^{i} \in v[\boldsymbol{A}]^{-1}\left(\psi^{i}\right)$ for any $\Delta \psi^{i} \in \operatorname{Ker}(\boldsymbol{A})$. Thus, by Eq. (18), $\psi^{i}+\Delta \psi^{i} \in \Psi^{i *}(\boldsymbol{A}, p)$. This holds for each consumer $i$, which satisfies the portfolio optimality condition from Definition 2 . The same holds true for the market clearing condition: $\mathbf{1} \in v[\boldsymbol{A}]^{-1}(\bar{\psi})$ if $\bar{\psi}-\mathbf{1} \in \operatorname{Ker}(\boldsymbol{A})$. Thus, the last condition in (15) is satisfied and the preimage $\pi^{-1}(L, A, p, c, \psi)$ is nonempty.

Property (iii) Note that $v[\boldsymbol{A}]$ is a linear mapping from $K$-dimensional space to $r$-dimensional space because $r=\operatorname{rank}(\boldsymbol{A})=\operatorname{rank}\left(\boldsymbol{A}^{\top}\right)$. Accordingly, $(\nu[\boldsymbol{A}])_{i=1}^{I}$ is a linear mapping from $I K$-dimensional space to $r I$-dimensional space. As part of $\pi$, all its arguments satisfy the $K$-dimensional market clearing condition $\bar{\psi}-\mathbf{1} \in\{0\}$ by Eq. (15). By contrast, restricted quasi-equilibria only satisfy the $r$-dimensional condition $\bar{\psi}-\mathbf{1} \in \operatorname{Ker}(\boldsymbol{A})$. Thus, the preimage of a quasiequilibrium portfolio vector under the restriction of $(v[\boldsymbol{A}])_{i=1}^{I}$ is of dimension $I K-I r-(K-r)=(I-1)(K-r)$.

Restricted quasi-equilibria are very well behaved: Demand, market prices, and best replies can be represented by differentiable bundle mappings at least in some neighborhood of the equilibrium. This is the content of the following three lemmata. To prepare for the endowment perturbations in the proof of Proposition 1, endowments $e$ are added as an explicit argument.

Lemma A10 For any $(\boldsymbol{A}, p, c, \psi) \in \hat{\boldsymbol{\Theta}}_{r}$ with $c \gg 0$, there are open neighborhoods $\mathcal{O} \subset \tilde{\mathcal{X}}_{r}$ of $(\boldsymbol{A}, p)$ and $\mathcal{E} \subset \mathbb{E}$ of $e$ as well as $C^{2}$ bundle mapping $\psi^{\Delta}: \mathcal{O} \times \mathcal{E} \rightarrow$ $\mathcal{V}\left(\tilde{\mathcal{A}}_{r}, \boldsymbol{A} \mapsto \operatorname{Im}\left(\boldsymbol{A}^{\top}\right)^{I}\right)$ that satisfies $\psi^{\triangle}[\boldsymbol{A}](p, e) \in \Psi^{*}(\boldsymbol{A}, p)$ on $\mathcal{O} \times \mathcal{E}$. 
Proof Since $U^{i}$ is strictly increasing under Assumption 1, the budget constraint in (1) holds with equality and the asset demand correspondence can be written as the preimage $\Psi^{i *}(\boldsymbol{A}, p)=A^{-1}\left(c_{1}^{i *}(\boldsymbol{A}, p)-e_{1}^{i}\right)$ under the linear transformation $A$ defined by $\boldsymbol{A}$. This implies that $v[\boldsymbol{A}] \circ \Psi^{i *}$ is single-valued. Moreover, it is continuous on $\mathcal{X}_{r}$ as $\Psi^{i *}$ is. Thus, the function $\psi^{i *}: \mathcal{X}_{r} \rightarrow \mathbb{R}^{K}$, defined as $\psi^{i *}=v[\boldsymbol{A}] \circ \Psi^{i *} \mid \mathcal{X}_{r}$, is a continuous selection. It must satisfy the first-order conditions of consumer $i$. Let $\tilde{\mathcal{Y}}_{r}$ be defined like $\mathcal{Y}_{r}$ in Eq. (15) but without the constraint $\bar{\psi}=\mathbf{1}$. Consider a bundle mapping $b: \tilde{\mathcal{Y}}_{r} \times \mathbb{E} \rightarrow \mathcal{V}\left(\tilde{\mathcal{A}}_{r}, \operatorname{Im}\left(\boldsymbol{A}^{\top}\right)^{I}\right)$ that covers id $\tilde{\mathcal{A}}_{r}$ and has components of the form

$$
b^{i}[\boldsymbol{A}](p, c, \psi, e)=D u^{i}\left[e_{\mathbf{1}}^{i}+\boldsymbol{A} \cdot \psi^{i}\right] \cdot \boldsymbol{A}-p .
$$

Note that $b^{i}[\boldsymbol{A}](p, c, \psi, e)=0$ represents the first-order conditions of consumer $i$ for any $c \gg 0$. The mapping $b$ is of class $C^{2}$ because $u^{i}$ is of class $C^{3}$ under Assumption 1. Its Jacobian with respect to $\psi^{i}$ in local coordinates is $D_{\psi^{i}} b^{i}[\boldsymbol{A}, p, c, \psi, e]=\boldsymbol{A}^{\top} \cdot D^{2} u^{i}\left[c^{i}\right] \cdot \boldsymbol{A}$, which is negative definite on $\operatorname{Im}\left(\boldsymbol{A}^{\top}\right)$. As a consequence, $d_{\psi^{i}} b^{i}[\boldsymbol{A}, p, c, \psi, e]$ is bijective for each consumer $i$, and therefore $d_{\psi} b[\boldsymbol{A}, p, c, \psi, e]$ is bijective as a whole. By Theorem A1, there exists a $C^{2}$ solution mapping $\psi^{\Delta}=\left(\psi^{1 \triangle}, \ldots, \psi^{I \triangle}\right)$ to the system of first-order conditions $b[\boldsymbol{A}]\left(p, c, \psi^{\triangle}[\boldsymbol{A}](p, e), e\right)=0$ for some open subset $\mathcal{O} \times \mathcal{E} \subset \tilde{\mathcal{X}}_{r} \times \mathbb{E}$.

A direct consequence of Lemma A10 is that optimal consumption $c^{\triangle}$, which is defined as

$$
c^{i \triangle}(\boldsymbol{A}, p, e)=e^{i}+\left(\begin{array}{c}
-p \\
\boldsymbol{A}
\end{array}\right) \cdot \psi^{i \Delta}(\boldsymbol{A}, p, e) \quad \forall i
$$

is a $C^{2}$ bundle mapping on $\mathcal{O} \times \mathcal{E}$ as well. This is also true for the market price function at regular restricted equilibria, but with $\mathcal{O}$ being a subset of $\tilde{\mathcal{A}}_{r}$ :

Lemma A11 For any $(\boldsymbol{A}, p, c, \psi) \in \hat{\boldsymbol{\Xi}}_{r}^{*}$ with $c \gg 0$, there are open neighborhoods $\mathcal{O} \subset \tilde{\mathcal{A}}_{r}$ of $\boldsymbol{A}$ and $\mathcal{E} \subset \mathbb{E}$ of $e$ as well as $C^{2}$ bundle mapping $p^{\triangle}: \mathcal{O} \times \mathcal{E} \rightarrow$ $\mathcal{V}\left(\tilde{\mathcal{A}}_{r}, \boldsymbol{A} \mapsto \operatorname{Im}\left(\boldsymbol{A}^{\top}\right)\right)$ that satisfies $p^{\triangle}[\boldsymbol{A}](e)=p^{*}(\boldsymbol{A})$ on $\mathcal{O} \times \mathcal{E}$. Its partial differential $d_{e} p^{\triangle}[\boldsymbol{A}, e]$ is surjective on $\mathcal{O} \times \mathcal{E}$.

Proof Let $\psi^{\triangle}$ be the $C^{2}$ function from Lemma A10. Note that the market clearing condition in Definition 2 implies the market clearing condition in Definition 6, and thus, the inverse demand function $p^{*}$ also solves $\bar{\psi}^{\triangle}[\boldsymbol{A}]\left(p^{*}(\boldsymbol{A}), e\right)=v[\boldsymbol{A}](\mathbf{1})$. To see that $p^{*}$ can be locally represented by a bundle mapping, consider again the mapping $b^{i}$ from Eq. (19). The Jacobian $D_{p} \psi^{i \triangle}[A, p, e]=\left(D_{\psi^{i}} b^{i}[A, p, c, \psi, e]\right)^{-1}$ is negative definite as the inverse of a negative definite matrix. As a consequence, $d_{p} \bar{\psi}^{\Delta}[\boldsymbol{A}, p, e]=$ $\sum_{i=1}^{I} d_{p} \psi^{i \triangle}[\boldsymbol{A}, p, e]$ is bijective since $D_{p} \bar{\psi}^{\Delta}[\boldsymbol{A}, p, e]=\sum_{i=1}^{I} D_{p} \psi^{i \triangle}[\boldsymbol{A}, p, e]$ is the sum of negative definite matrices. By Theorem $\mathrm{A} 1$, there exists a unique $C^{2}$ solution $\psi^{\Delta}$ to

$$
\bar{\psi}^{\triangle}[\boldsymbol{A}]\left(p^{\triangle}[\boldsymbol{A}](e), e\right)=v[\boldsymbol{A}](\mathbf{1}) ;
$$


for some open subset $\mathcal{O} \times \mathcal{E} \subset \tilde{\mathcal{A}}_{r} \times \mathbb{E}$. By uniqueness of the solution, it must agree with $p^{*}$. By the chain rule, $D_{e} p^{\triangle}[\boldsymbol{A}, e]=-\left(D_{p} \bar{\psi}^{\triangle}[\boldsymbol{A}, p, e]\right)^{-1} \cdot D_{e} \bar{\psi}^{\triangle}[\boldsymbol{A}, p, e]$, and since

$$
D_{e} \bar{\psi}^{\Delta}[\boldsymbol{A}, p, e]=-\sum_{i=1}^{I}\left(D_{\psi^{i}} b^{i}[\boldsymbol{A}, p, c, \psi, e]\right)^{-1} \cdot D_{e^{i}} b^{i}[\boldsymbol{A}, p, c, \psi, e]
$$

is surjective because $D_{e_{\mathbf{1}}^{i}} b^{i}[\boldsymbol{A}, p, c, \psi, e]=\boldsymbol{A}^{\top} \cdot D^{2} u^{i}\left[c^{i}\right]$ is, the proof is completed.

The following lemma shows that the best-reply correspondence can be locally equipped with the structure of a bundle mapping:

Lemma A12 For any $\xi \in \hat{\Xi}_{r}^{*}$ and $\left(L_{\#}, A_{\#}, p_{\#}, c_{\#}, \psi_{\#}\right)=\pi(\xi)$, there is some open subset $\mathcal{O} \times \mathcal{E} \subset \mathcal{V}\left(\mathcal{G}_{r}(|\boldsymbol{\Omega}|), L \mapsto L^{K}\right) \times \mathbb{E}$ containing $\left(L_{\#}, \boldsymbol{A}_{\#}, e_{\#}\right)$ and a $C^{1}$ bundle mapping $A^{\triangle}: \mathcal{O} \times \mathcal{E} \rightarrow \mathcal{V}\left(\mathcal{G}_{r}(|\boldsymbol{\Omega}|), L \mapsto L^{K}\right)$ such that $A^{\triangle}[L](\boldsymbol{A}, e)=\hat{A}^{*}(\boldsymbol{A})$ on $\mathcal{O} \times \mathcal{E}$.

Proof Define $A^{\triangle}(L, \boldsymbol{A}, e)=\left(L, \hat{A}^{*}(\boldsymbol{A})\right)$, which is of class $C^{1}$ on an open set $\mathcal{O} \times \mathcal{E}$; otherwise, $\hat{A}^{*}$ could not be continuously differentiable at $\boldsymbol{A}_{\#}$, Definition 3 would be violated, and the reference equilibrium $\xi$ could not be regular. It remains to be verified that $\hat{A}^{*}(\boldsymbol{A}) \in L^{K}$ holds for all $(\boldsymbol{A}, e) \in \mathcal{O} \times \mathcal{E}$. Suppose not; then, $\hat{A}^{k *}(\boldsymbol{A}) \notin \operatorname{Im}\left(\boldsymbol{A}_{\#}^{\neg k}\right)$ for some producer $k$ because $L=\operatorname{Im}(A)$ at any restricted quasi-equilibrium. By (14), this is only possible if $\hat{\mathcal{A}}_{r}^{k}$ has a discontinuity at $\boldsymbol{A}$. However, in that case $\hat{A}^{k *}$ would be discontinuous at $\boldsymbol{A}$, and $A^{\triangle}$ could not be of class $C^{1}$.

With the help of these lemmata, the proof of the main theorem is straightforward. Proof of Theorem 1 Fix some $r \in \mathbb{N}$ and let $(\boldsymbol{A}, p, c, \psi) \in \Xi_{r}^{* *}$; then, $\xi=$ $\pi(\boldsymbol{A}, p, c, \psi)$ is a restricted quasi-equilibrium by Lemma A9. Let $\mathcal{O}$ be an open neighborhood of $\xi$ in $\mathcal{Z}_{r}$, and let $\mathcal{E}$ be an open neighborhood of the endowment vector $e$ in $\mathbb{E}$. Define a bundle mapping $f: \mathcal{O} \times \mathcal{E} \rightarrow \mathcal{Z}_{r}$ that covers the identity as

$$
f[L](\boldsymbol{A}, p, c, \psi, e)=\left(\begin{array}{c}
A^{\triangle}[L, \boldsymbol{A}](e)-\boldsymbol{A} \\
p^{\triangle}[\boldsymbol{A}](e)-p \\
c^{\triangle}[\boldsymbol{A}](p, e)-c \\
\psi^{\triangle}[\boldsymbol{A}](p, e)-\psi
\end{array}\right) .
$$

Note that $A^{\triangle}, p^{\triangle}, c^{\triangle}$, and $\psi^{\triangle}$ are (twice) continuously differentiable by Lemmata A10, A11, A12 and Eq. (20). Define $f_{e}[L](\boldsymbol{A}, p, c, \psi)=f[L](\boldsymbol{A}, p, c, \psi, e)$ as the restriction for fixed $e$. By construction, $f_{e}\left(\xi^{\prime}\right)=0$ is equivalent to $\pi^{-1}\left(\xi^{\prime}\right) \in \hat{\Xi}_{r}^{*}$. The Jacobian $D f_{e}\left[\xi^{\prime}\right]=D f\left[\xi^{\prime}, e\right]$ at any such $\xi^{\prime}$, with brackets omitted for clarity, is of the form

$$
D_{(\boldsymbol{A}, p, c, \psi)} f[L, \boldsymbol{A}, p, c, \psi, e]=\left(\begin{array}{cccc}
D_{A} A^{\triangle}-\boldsymbol{I} & \mathbf{0} & \mathbf{0} & \mathbf{0} \\
D_{\boldsymbol{A}} p^{\triangle} & -\boldsymbol{I} & \mathbf{0} & \mathbf{0} \\
D_{\boldsymbol{A}} c^{\triangle} & D_{p} c^{\triangle} & -\boldsymbol{I} & \mathbf{0} \\
D_{\boldsymbol{A}} \psi^{\triangle} & D_{p} \psi^{\triangle} & \mathbf{0} & -\boldsymbol{I}
\end{array}\right),
$$


which is a lower triangular block matrix. By regularity, $D_{A} A^{\triangle}[\boldsymbol{A}, e]-\boldsymbol{I}$ has full rank according to Definition 3; otherwise, $d \hat{A}^{*}[\boldsymbol{A}]-\mathrm{id}_{L^{K}}$ would not span $L^{K}=\operatorname{Im}(\boldsymbol{A})^{K}$. Thus, all matrices along the main diagonal are invertible, and by Lemma A5, $f_{e} \pitchfork \sigma_{0}\left(\mathcal{G}_{r}(|\boldsymbol{\Omega}|)\right)$. By the preimage theorem, $\operatorname{dim}\left(f_{e}^{-1}\left(\sigma_{0}\left(\mathcal{G}_{r}(|\boldsymbol{\Omega}|)\right)\right)\right)=$ $\operatorname{dim}\left(\sigma_{0}\left(\mathcal{G}_{r}(|\boldsymbol{\Omega}|)\right)\right)=\operatorname{dim}\left(\mathcal{G}_{r}(|\boldsymbol{\Omega}|)\right)$. Since all financial indeterminacy is annulled at a restricted quasi-equilibrium, this dimension measures only the degree of real indeterminacy, which is therefore $r(|\boldsymbol{\Omega}|-r)$ by Lemma A2. Furthermore, as $\pi$ is a submersion by Lemma A9, $f_{e} \pitchfork \sigma_{0}\left(\mathcal{G}_{r}(|\boldsymbol{\Omega}|)\right)$ implies $f_{e} \circ \pi \pitchfork \sigma_{0}\left(\mathcal{G}_{r}(|\boldsymbol{\Omega}|)\right)$. Another application of the preimage theorem reveals that $\left(f_{e} \circ \pi\right)^{-1}\left(\sigma_{0}\left(\mathcal{G}_{r}(|\boldsymbol{\Omega}|)\right)\right)$ is a $C^{1}$ manifold of dimension $r(|\boldsymbol{\Omega}|-r)+(I-1)(K-r)$, in which the difference in dimensions $(I-1)(K-r)$ follows from the third property of Lemma A9. As this argument can be repeated for any $\xi \in \hat{\Xi}_{r}^{*}$, it can be concluded that $\hat{\Xi}_{r}^{*}$ in its entirety is a $C^{1}$ manifold of the computed dimension or the empty set. Since $\Xi_{r}^{* *}$ is an open subset of $\hat{\Xi}_{r}^{*}$ by Lemma A8, the theorem is proven.

Proof of Corollary 1 Recall that $\Xi_{r}^{* *} \subseteq \Xi_{r}^{*}$. The dimension of $\Xi_{r}^{*}$ has an upper bound: The set of equilibrium asset spans is at most of $\operatorname{dim}\left(\mathcal{G}_{r}(|\boldsymbol{\Omega}|)\right)=r(|\boldsymbol{\Omega}|-r)$ by Lemma A2. For each asset span $L \in \mathcal{G}_{r}(|\boldsymbol{\Omega}|)$, equilibrium asset payoffs $\boldsymbol{A} \in L^{K}$ are isolated points. If not, there would exist some nonzero $\Delta \boldsymbol{A} \in L^{K}$ such that $d\left(A^{*}(\boldsymbol{A})-\boldsymbol{A}\right)[\boldsymbol{A}](\Delta \boldsymbol{A})=0$, but this is ruled out by the third condition of Definition 3. In fact, there can only be a finite number of isolated points because $\mathcal{A}$ is compact under Assumption 3. Furthermore, for each equilibrium asset payoff matrix $\boldsymbol{A}$, there is a unique equilibrium price vector by Lemma A11. Finally, for each tuple $(\boldsymbol{A}, p) \in \tilde{\mathcal{X}}_{r}$, there is a unique equilibrium consumption vector $c$ and up to $(I-r)(K-r)$ consistent portfolio vectors $\psi$. Thus, the upper bound is $\operatorname{dim}\left(\Xi_{r}^{*}\right)=r(|\boldsymbol{\Omega}|-r)+(I-1)(K-r)$. Since $\operatorname{dim}\left(\Xi_{r}^{* *}\right)=r(|\boldsymbol{\Omega}|-r)+(I-1)(K-r)$ by Theorem 1, the upper bound is reached.

Proof of Corollary 2 It must be shown that the projection of $\Xi_{r}^{* *}$ onto the Grassmannian $\mathcal{G}_{r}(|\boldsymbol{\Omega}|)$ is an open subset. To do so, define the smooth function $h: \pi\left(\hat{\boldsymbol{\Xi}}_{r}^{*}\right) \rightarrow \mathcal{G}_{r}(|\boldsymbol{\Omega}|)$ as $h(L, \boldsymbol{A}, p, c, \psi)=L$. Recall from the proof of Theorem 1 that $\xi \in \pi\left(\hat{\boldsymbol{\Xi}}_{r}^{*}\right)$ is equivalent to $f_{e}(\xi)=0$. Therefore, $h^{-1}(L)$ and $f_{e}[L]^{-1}(0)$ are equipotent. Since the Jacobian in (23) has full rank, $f_{e}[L] \pitchfork\{0\}$ and the preimage theorem implies that $f_{e}[L]^{-1}(0)$ is a 0 -dimensional $C^{1}$ manifold. By equipotence, $h^{-1}(L)$ is always a set of isolated points, and thus, $h$ is locally injective. Since domain and range of $h$ are manifolds of equal dimension (see Theorem 1 and Lemma A2), $h$ is open by invariance of domain. Finally, recall that $\pi$ is a submersion. By Lemma A8, $\Xi_{r}^{* *}$ is an open subset of $\hat{\Xi}_{r}^{*}$, and thus, $h\left(\pi\left(\Xi_{r}^{* *}\right)\right)$ is an open subset of the Grassmannian.

Finally, it is time to prove that every open subset of $\Xi_{r}^{* *}$ contains two equilibria that can be welfare-ranked:

Proof of Proposition 1 Suppose not; then, for some $r \in \mathbb{N}$ there would be a welfare level $w \in \mathbb{R}$ and an open subset $\mathcal{S} \subseteq \Xi_{r}^{* *}$ with the property that $W(\xi)=w \forall \xi \in \mathcal{S}$. That is to say, $D W[\xi] \perp T \Xi_{r}^{* *}[\xi] \forall \xi \in \mathcal{S}$. Since the tangent space to $\Xi_{r}^{* *}$ at $\xi$ satisfies $T \Xi_{r}^{* *}[\xi]=\operatorname{Ker}\left(d f_{e}[\pi(\xi)] \circ d \pi[\xi]\right)$, this orthogonality condition is equivalent to $\operatorname{Ker}\left(d f_{e}[\pi(\xi)] \circ d \pi[\xi]\right) \subseteq \operatorname{Ker}(d W[\xi]) \forall \xi \in \mathcal{S}$. As in the proof of Theorem 1, let 
$\mathcal{O} \times \mathcal{E}$ be an open subset of $\mathcal{Z}_{r} \times \mathbb{E}$. Now, consider the function $g: \mathcal{O} \times \mathcal{E} \rightarrow \mathcal{Z}_{r} \times \mathbb{R}$, defined as

$$
g(L, \boldsymbol{A}, p, c, \psi, e)=\left(\begin{array}{c}
f(L, \boldsymbol{A}, p, c, \psi, e) \\
W(\boldsymbol{A}, p, c, \psi)
\end{array}\right)
$$

with restriction $g_{e}(L, \boldsymbol{A}, p, c, \psi)=g(L, \boldsymbol{A}, p, c, \psi, e)$ for fixed $e$. The Jacobian of $g$ in local coordinates has the form

$D_{(\boldsymbol{A}, p, c, \psi, e)} g[L, \boldsymbol{A}, p, c, \psi, e]=\left(\begin{array}{ccccc}D_{A} A^{\Delta}-\boldsymbol{I} & \mathbf{0} & \mathbf{0} & \mathbf{0} & D_{e} A^{\Delta} \\ D_{A} p^{\triangle} & -\boldsymbol{I} & \mathbf{0} & \mathbf{0} & D_{e} p^{\triangle} \\ D_{A} c^{\triangle} & D_{p} c^{\triangle} & -\boldsymbol{I} & \mathbf{0} & D_{e} c^{\Delta} \\ D_{A} \psi^{\triangle} & D_{p} \psi^{\triangle} & \mathbf{0} & -\boldsymbol{I} & D_{e} \psi^{\Delta} \\ 0 & D_{A} W & 0 & D_{c} W & 0\end{array}\right)$,

in which all brackets are omitted for the sake of clarity. It is easy to check that $g \pitchfork\{0\} \times\{w\}:$ As shown above, the entire block above the last row has full rank at any equilibrium, and the last row of $g$ can be perturbed independently. To see this, note that $d_{e} p^{\triangle}[\boldsymbol{A}, e]$ is surjective by Lemma A10 but its kernel is of dimension nullity $\left(d_{e} p^{\triangle}[\boldsymbol{A}, e]\right) \geq I(|\boldsymbol{\Omega}|+1)-K$. Any vector $v \in \operatorname{Ker}\left(d_{e} p^{\triangle}[\boldsymbol{A}, e]\right)$ satisfies $D_{e} p^{\triangle}[\boldsymbol{A}, e] \cdot v=0$, and thus, $D_{e} A^{\triangle}[\boldsymbol{A}, e] \cdot v=0$, but $D_{e} c^{\triangle}[\boldsymbol{A}, p, e] \cdot v \neq 0$ and potentially $D_{e} \psi^{\Delta}[\boldsymbol{A}, p, e] \cdot v \neq 0$. These two effects can be undone by changes in $c$ and $\psi$, and changes in $c$ perturb $W$. In other words, the above matrix has full rank and $g \pitchfork \sigma_{0}\left(\mathcal{G}_{r}(|\boldsymbol{\Omega}|)\right) \times\{w\}$. By the parametric transversality theorem (see Hirsch (1994), p. 79-80, Theorem 2.7 and Guillemin and Pollack (1974), p. 68), $g_{e} \pitchfork \sigma_{0}\left(\mathcal{G}_{r}(|\boldsymbol{\Omega}|)\right) \times\{w\}$ for any $e \in \mathcal{E}_{*}$, which is an open, dense, full-measure subset of $\mathcal{E}$. As this argument can be repeated for any economy, the statement can be extended to an open, dense, full-measure subset $\mathbb{E}_{*} \subseteq \mathbb{E}$. As a consequence, $\operatorname{rank}\left(d f_{e}[\pi(\xi)] \circ d \pi[\xi]\right)=\operatorname{rank}\left(d g_{e}[\pi(\xi)] \circ d \pi[\xi]\right)-1$, and by the rank-nullity theorem (see Lang (2004), p. 61, Theorem 3.2), nullity $\left(d f_{e}[\pi(\xi)] \circ d \pi[\xi]\right)=$ nullity $\left(d g_{e}[\pi(\xi)] \circ d \pi[\xi]\right)+1$. But since $\operatorname{Ker}\left(d f_{e}[\pi(\xi)] \circ d \pi[\xi]\right) \subseteq \operatorname{Ker}(d W[\xi])$ requires nullity $\left(d f_{e}[\pi(\xi)] \circ d \pi[\xi]\right)=\operatorname{nullity}\left(d g_{e}[\pi(\xi)] \circ d \pi[\xi]\right)$, this contradicts the initial statement, which proves the proposition.

The appendix ends with proofs of the two claims from Sect. 6.

Proof of Claim 1 Consider a pseudo-economy in which a complete set of (two) statecontingent claims is available in addition to the assets of both producers. In this pseudo-economy, the asset span is independent of the production plans chosen, and there is a unique state price vector $q$ that clears the market. The pseudo-price of the $k^{\text {th }}$ asset,

$$
\tilde{p}^{k}=q \cdot A^{k}
$$

can at any stage of replication $n$ be written as a function $\tilde{p}_{n}^{k}\left(\alpha^{k}, \alpha^{\neg k}\right)$ of two scalars. The latter parameter is the average choice of all other producers $\alpha^{\neg k}=\frac{1}{2 n-1} \sum_{j \neq k} \alpha^{j}$. 
Since all production sets are identical, $\alpha^{\neg k} \in[-1 / 2,1 / 2]$. Moreover, $\tilde{p}_{n}^{k}\left(\alpha^{k}, \alpha^{-k}\right)$ equivalently represents the pseudo-price of asset $k$ if all other $2 n-1$ producers chose the identical strategy $\alpha^{-k}$. Therefore, it can be determined by solving the system of Arrow-Debreu equilibrium conditions

$$
\begin{aligned}
q-D u^{i}\left[c_{\mathbf{1}}^{i}\right] & =0 \quad \forall i \\
q \cdot\left(c_{\mathbf{1}}^{i}-e_{\mathbf{1}}^{i}\right)-\left(c_{0}^{i}-e_{0}^{i}\right) & =0 \quad \forall i \\
\sum_{i=1}^{2 n}\left(c_{\mathbf{1}}^{i}-e_{\mathbf{1}}^{i}\right)-\left(\begin{array}{l}
n / 2+\alpha^{k}+(2 n-1) \alpha^{\neg k} \\
n / 2-\alpha^{k}-(2 n-1) \alpha^{\neg k}
\end{array}\right) & =0
\end{aligned}
$$

jointly with (24). The solution is

$$
\tilde{p}_{n}^{k}\left(\alpha^{k}, \alpha^{\neg k}\right)=\frac{n+2 n \alpha^{k}}{\alpha^{k}-\alpha^{\neg k}+n+2 n \alpha^{\neg k}}-\frac{n-2 n \alpha^{k}}{\alpha^{k}-\alpha^{\neg k}-\frac{3}{2} n+2 n \alpha^{\neg k}},
$$

and its second-order derivative with respect to $\alpha^{k}$ is

$$
4 n\left(\frac{\alpha^{\neg k}+\frac{1}{2}-n\left(2 \alpha^{\neg k}+1\right)}{\left(\alpha^{k}-\alpha^{\neg k}+n\left(2 \alpha^{\neg k}+1\right)\right)^{3}}+\frac{8 \alpha^{\neg k}-4+n\left(12-16 \alpha^{\neg k}\right)}{\left(2 \alpha^{k}-2 \alpha^{\neg k}+n\left(4 \alpha^{\neg k}-3\right)\right)^{3}}\right) .
$$

It is easy to verify that for any combination $\alpha^{k}, \alpha^{k} \in(-1 / 2,1 / 2)$, the left fraction has a negative numerator and a positive denominator, and the right fraction has a positive numerator and a negative denominator. As a result, $D_{\alpha^{k}}^{2} \tilde{p}_{n}^{k}\left[\alpha^{k}, \alpha^{\neg k}\right]<0 \forall n \geq$ 1 and since $\kappa^{k}=0$, the payoff function of the producer in the pseudo-economy is strictly concave. As all production sets are nonempty, convex, and compact, this is sufficient to guarantee the existence of an equilibrium for the pseudo-economy. By symmetry of the game, at least one equilibrium must involve symmetric strategies. By construction,

$$
p_{n}^{k *}\left(\alpha^{k}, \alpha^{\neg k}\right)= \begin{cases}\beta\left(\alpha^{\neg k}\right) & \text { if } \alpha^{k}=\alpha^{\neg k} \\ \tilde{p}_{n}^{k *}\left(\alpha^{k}, \alpha^{\neg k}\right) & \text { otherwise }\end{cases}
$$

in which $\beta$ is the solution for $p^{\neg k}$ in the system of incomplete-market equilibrium conditions

$$
\begin{aligned}
p^{\neg k}-D u^{i}\left[e_{\mathbf{1}}^{i}+\boldsymbol{A}^{\neg k} \cdot \psi_{\neg k}^{i}\right] \cdot \boldsymbol{A}^{\neg k} & =0 \quad \forall i \\
\sum_{i=1}^{2 n} \psi_{\neg k}^{i}-2 n & =0 \\
\boldsymbol{A}^{\neg k}-\left(\begin{array}{c}
1 / 2+\alpha^{\neg k} \\
1 / 2-\alpha^{\neg k}
\end{array}\right) & =0,
\end{aligned}
$$


which can be solved by setting $n=1$ since $n$ drops out of the market clearing equation because all consumers of a type choose identical portfolios. The solution is

$$
\beta\left(\alpha^{\neg k}\right)=\frac{15-24 \alpha^{\neg k}+\sqrt{33-\left(80-64 \alpha^{\neg k}\right) \alpha^{\neg k}}}{12-16 \alpha^{\neg k}} .
$$

Furthermore, $n$ drops out of the pseudo-price equation in the case of symmetric strategies:

$$
\tilde{p}_{n}^{k *}\left(\alpha^{\neg k}, \alpha^{\neg k}\right)=\frac{5-8 \alpha^{\neg k}}{3-4 \alpha^{\neg k}}
$$

Since $\beta\left(\alpha^{\neg k}\right)>\tilde{p}_{n}^{k *}\left(\alpha^{\neg k}, \alpha^{\neg k}\right)$ for any $\alpha^{\neg k} \in(-1 / 2,1 / 2)$, the existence of a symmetric equilibrium for the pseudo-economy implies the existence of a symmetric equilibrium for the true economy.

Proof of Claim 2 Taking the limit of (25) results in $\lim _{n \rightarrow \infty} \tilde{p}_{n}^{k *}\left(\alpha^{k}, \alpha^{-k}\right)=\gamma\left(\alpha^{\neg k}\right)+$ $\delta\left(\alpha^{\natural}\right) \alpha^{k}$, in which $\gamma$ and $\delta$ are defined as

$$
\gamma\left(\alpha^{\neg k}\right)=\frac{5}{3+\left(2-8 \alpha^{\neg k}\right) \alpha^{\neg^{k}}}, \quad \delta\left(\alpha^{\neg k}\right)=\frac{2-16 \alpha^{\neg k}}{3+\left(2-8 \alpha^{\neg k}\right) \alpha^{\neg^{k}}},
$$

and the statement follows from (26).

\section{References}

Allen, B.: Randomization and the limit points of monopolistic competition. J. Math. Econ. 23, 205-218 (1994)

Balasko, Y., Cass, D.: The structure of financial equilibrium with exogenous yields: the case of incomplete markets. Econometrica 57, 135-162 (1989)

Bejan, C.: The objective of a privately owned firm under imperfect competition. Econ. Theory 37, 99-118 (2008). https://doi.org/10.1007/s00199-007-0289-5

Böhm, V.: The foundation of the theory of monopolistic competition revisited. J. Econ. Theory 63, 208-218 (1994)

Carvajal, A., Rostek, M., Weretka, M.: Competition in financial innovation. Econometrica 80, 1895-1936 (2012)

Cournot, A.: Recherches sur les Principes Mathématiques de la Théorie des Richesses. Hachette, Paris (1838)

Demichelis, S., Ritzberger, K.: A general equilibrium analysis of corporate control and the stock market. Econ. Theory 46, 221-254 (2011). https://doi.org/10.1007/s00199-009-0511-8

Diamond, P.A.: The role of a stock market in a general equilibrium model with technological uncertainty. Am. Econ. Rev. 57, 759-776 (1967)

Dierker, E.: Two remarks on the number of equilibria of an economy. Econometrica 40, 951-953 (1972)

Dierker, E., Dierker, H.: Welfare and efficiency in incomplete market economies with a single firm. J. Math. Econ. 46, 652-665 (2010)

Dierker, H., Grodal, B.: Non-existence of Cournot-Walras equilibrium in a general equilibrium model with two oligopolists. In: Hildenbrand, W., Mas-Colell, A. (eds.) Contributions to Mathematical Economics, pp. 167-185. North Holland, Amsterdam (1986)

Dierker, E., Grodal, B.: The price normalization problem in imperfect competition and the objective of the firm. Econ. Theory 14, 257-284 (1999). https://doi.org/10.1007/s001990050293 
Drèze, J.: Investment under private ownership: optimality, equilibrium and stability. In: Drèze, J. (ed.) Allocation Under Uncertainty, Equilibrium and Optimality, pp. 129-165. Wiley, New York (1974)

Duffie, D., Shafer, W.: Equilibrium in incomplete markets: I. A basic model of generic existence. J. Math. Econ. 14, 285-300 (1985)

Faias, M.: Approximate equilibrium in pure strategies for a two-stage game of asset creation. Decis. Econ. Financ. 31, 117-136 (2008)

Gabszewicz, J.J., Vial, J.P.: Oligopoly à la Cournot in general equilibrium analysis. J. Econ. Theory 4, 381-400 (1972)

Geanakoplos, J., Mas-Colell, A.: Real indeterminacy with financial assets. J. Econ. Theory 47, 22-38 (1989)

Govaerts, W.: Defining functions for manifolds of matrices. Linear Algebra Appl. 266, 49-68 (2014)

Guillemin, V., Pollack, A.: Differential Topology. Prentice-Hall, Englewood Cliffs (1974)

Harsanyi, J.C.: Oddness of the number of equilibrium points: a new proof. Int. J. Game Theory 2, 235-350 (1973)

Hart, O.D.: On the optimality of equilibrium when the market structure is incomplete. J. Econ. Theory 11, 418-443 (1975)

Hens, T., Pilgrim, B.: General Equilibrium Foundations of Finance: Structure of Incomplete Market Models. Springer, Dodrecht (2002)

Hirsch, M.W.: Differential Topology, 5th edn. Springer, Berlin (1994)

Hirsch, M.D., Magill, M., Mas-Colell, A.: A geometric approach to a class of equilibrium existence theorems. J. Math. Econ. 19, 95-106 (1990)

Husseini, S.Y., Lasry, J.M., Magill, M.J.: Existence of equilibrium with incomplete markets. J. Math. Econ. 19, 39-67 (1990)

Lang, S.: Linear Algebra, 3rd edn. Springer, New York (2004)

Magill, M., Quinzii, M.: Theory of Incomplete Markets. MIT Press, Cambridge (1996)

Mas-Colell, A.: Indeterminacy in incomplete market economies. Econ. Theory 1, 45-61 (1991). https:// doi.org/10.1007/BF01210573

Milne, F., Ritzberger, K.: Strategic pricing of equity issues. Econ. Theory 20, 271-294 (2002). https://doi. org/10.1007/s001990100212

Nagata, R.: The degree of indeterminacy of equilibria with incomplete markets. J. Math. Econ. 29, 109-123 (1998)

Novshek, W., Sonnenschein, H.: Cournot and Walras equilibria. J. Econ. Theory 19, 223-266 (1978)

Novshek, W., Sonnenschein, H.: Walrasian equilibria as limits of noncooperative equilibria. Part ii: pure strategies. J. Econ. Theory 30, 171-187 (1983)

Reny, P.J.: Introduction to the symposium on discontinuous games. Econ. Theory 61, 423-429 (2016). https://doi.org/10.1007/s00199-016-0962-7

Roberts, K.: The limit points of monopolistic competition. J. Econ. Theory 22, 256-278 (1980)

Shirai, K.: An existence theorem for Cournot-Walras equilibria in a monopolistically competitive economy. J. Math. Econ. 46, 1093-1102 (2010)

Villanacci, A., Carosi, L., Benevieri, P., Battinelli, A.: Differential Topology and General Equilibrium with Complete and Incomplete Markets. Kluwer Academic Publishers, Boston (2002)

Werner, J.: Structure of financial markets and real indeterminacy of equilibria. J. Math. Econ. 19, 113-151 (1990)

Zierhut, M.: Constrained efficiency versus unanimity in incomplete markets. Econ. Theory 64, 23-45 (2017). https://doi.org/10.1007/s00199-016-0968-1

Zierhut, M.: Nonexistence of constrained efficient plans. J. Math. Econ. 83, 127-136 (2019)

Zierhut, M.: Generic regularity of differentiated product oligopolies. Econ. Theory (forthcoming) (2020)

Publisher's Note Springer Nature remains neutral with regard to jurisdictional claims in published maps and institutional affiliations. 